\title{
Le lexique des lieux de culte dans la Périégèse de Pausanias*
}

\author{
Vinciane Pirenne-Delforge
}

Quiconque a déjà étudié l'un ou l'autre aspect de cet ensemble complexe qu'est la religion des anciens Grecs n'a sans doute pas échappé à la consultation de la Périégèse de Pausanias. Cette œuvre du $\mathrm{II}^{\mathrm{e}}$ siècle de notre ère est une source fondamentale pour appréhender le paysage religieux de la Grèce. Toutefois, le cadre chronologique de ce texte - à savoir la période romaine impériale - impose une sorte de doute méthodique quant à l'ancienneté des données qui s'y trouvent. Un autre problème, inhérent à l'œuvre elle-même, vient doubler celui de la chronologie: Pausanias est volontairement sélectif et il opère des choix qui livrent une image forcément tronquée des réalités du terrain. À cela s'ajoute une utilisation problématique de la Périégèse par ses lecteurs modernes. Ainsi, la consultation ponctuelle et souvent morcelée de ce vaste ensemble ne permet guère de mesurer l'exacte portée des informations obtenues.

Pour affiner l'analyse, il convient de replacer la Périégèse dans son contexte romain, de tenir compte du caractère sélectif des descriptions proposées, mais aussi de prendre en compte l'ensemble des données d'un même type au fil des dix livres rédigés par l'érudit voyageur. Pour ce dernier point, l'étude du lexique religieux est une option intéressante : elle permet d'éviter le caractère aléatoire des choix qui ne seraient guidés que par une nécessité particulière et elle contraint l'interprète à tenir compte de la totalité de l'œuvre.

Dans cette perspective, le vocabulaire est devenu le fil rouge d'une enquête menée sur les relations entre Pausanias et ce que l'on appelle conventionnellement " la religion grecque " : la représentation du monde des dieux, la description des statues, des sacrifices, des fêtes, ainsi que l'évocation des cultes à mystères ont été abordées en partant du vocabulaire employé par Pausanias. ${ }^{I}$ Un tel lexique religieux comprend aussi le vocabulaire varié servant à désigner les lieux de culte. Or la Périégèse compte des centaines de sanctuaires qui sont tantôt simplement mentionnés en passant, tantôt décrits avec plus ou moins de détails selon les cas.

* Cette étude a bénéficié de la lecture et des conseils de Pierre Bonnechere (Université de Montréal) et de Gérald Purnelle (Université de Liège). Qu’ils en soient tous deux remerciés. Je suis également très reconnaissante à Fernande et Tonio Hölscher de m’avoir associée à ce volume de l'Archiv für Religionsgeschichte.

I V. Pirenne-Delforge, Retour à la source. Pausanias et la religion grecque, Kernos supplément 20 (2008). 
Dès lors, l'ambition de cet article est de fournir le cadre "interne » de l'utilisation du vocabulaire des lieux de culte dans l'œuvre de Pausanias. Il s'agit de mettre en perspective l'ensemble des occurrences de ce lexique pour en déduire des lignes de force. On pourra peut-être s'étonner de l'absence de recours systématique aux fouilles archéologiques menées sur certains des sites que compte la description de Pausanias. Ce choix est délibéré pour d'évidentes raisons pratiques : la confrontation avec le terrain reviendrait à produire un commentaire archéologique systématique de la Périégèse, ce qui est un autre travail.

Au-delà des raisons pratiques, toutefois, ce choix relève surtout d'un impératif de méthode. En effet, le risque de raisonnement circulaire qu'impliquerait une telle démarche n'est pas mince. Bon nombre de fouilles sur le continent grec ont été guidées par les comptes rendus des visites de Pausanias et bien des structures ont été identifiées en fonction des renseignements qu’il a livrés. En privilégiant l'analyse interne du lexique, on peut espérer sortir des cercles vicieux de l'identification, fondée sur le texte de Pausanias, d'une structure mise au jour et contribuant, à son tour, à donner son sens au vocabulaire utilisé par Pausanias pour désigner ladite structure ... Cependant, il est clair qu'une telle adéquation est parfois possible et a donné de bons résultats. ${ }^{2}$ On ne se privera dès lors pas de faire parfois appel aux données du terrain lorsque les certitudes d'une identification ne reposeront pas sur le seul Pausanias. Mais au-delà de l'éventuel ancrage matériel de l'argumentation, l'objectif du présent article est de fournir une base de travail qui permette à d'autres d'élargir l'enquête aux données de terrain et d'affiner les résultats de la présente analyse interne du lexique des lieux de culte. ${ }^{3}$

La langue grecque dispose d'un vocabulaire varié pour désigner les lieux du culte. ${ }^{4}$ Pour rendre compte de ces réalités qui hantent les lieux visités, Pausanias suit le principe directeur général de sa présentation de "ce qu'il faut voir " : ${ }^{5}$ il sélectionne l'information qui lui semble digne d'intérêt et de mémoire, sans s'arrêter toujours à une description précise. Cela ne manque pas de poser bien des problèmes à l'interprète moderne.

2 Un exemple parmi d'autres : le « megaron » de Lykosoura. Voir M. Jost, Sanctuaires et cultes d'Arcadie (1985) I77 et fig. I-3, pl. 46.

3 Les comptages qui apparaissent dans la suite sont fondés sur l'index lemmatisé de la Périégèse réalisé au Laboratoire d'Analyse Statistique des Langues Anciennes (LASLA) de l'Université de Liège : V. Pirenne-Delforge - G. Purnelle, Pausanias, Periegesis. Index verborum, Liste de fréquence, Index nominum (1997). Cet index se fonde sur l'édition en trois volumes, parus chez Teubner, de M. Rocha-Pereira, Pausanias Graeciae Descriptio (1989-1990²).

4 Cf. Pollux I, 6-Io. - M. Morani, "Sull'espressione linguistica dell'idea di (santuario» nelle civiltà classiche", in : M. Sordi (éd.), Santuari e politica nel mondo antico (1983) 5-32; M. Casevitz, "Temples et sanctuaires : ce qu'apprend l'étude lexicologique », in : G. Roux (éd.), Temples et sanctuaires. Séminaire de recherche I98I-1983 (1984) 8I-95.

Paus. I, 39, 3 ; III, II, I. 
Pausanias utilise, pour ce type de description, une bonne part des ressour-

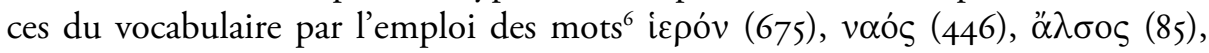

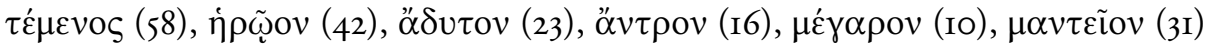

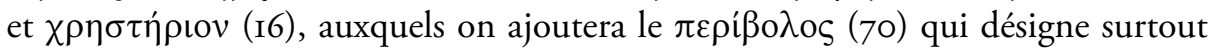
l'enclos des sanctuaires. ${ }^{7}$ On sera amenée à nuancer ces chiffres en cours de route car toutes les occurrences de certains termes n'ont pas nécessairement une portée religieuse et certaines occurrences entrent dans des citations et non dans des descriptions.

\section{Le sanctuaire en ses termes génériques}

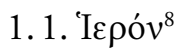

Depuis l'époque classique, la forme substantivée $\tau o ̀$ ífóv désigne le sanctuaire dans son acception la plus générale et la plus vague. ${ }^{9}$ Le caractère sacré du lieu prédomine dans l'emploi de ce terme pouvant recouvrir des réalités très diverses. Il sert donc admirablement les buts de Pausanias qui y recourt très fréquemment. Quand il ne l'utilise pas simplement au pluriel comme générique de différents lieux consacrés, ${ }^{10}$ les emplois sont divers : (I) le hieron apparaît en alternance avec d'autres termes désignant diverses réalités religieuses dans une séquence simplement énonciative - des préoccupations stylistiques guident alors le choix de son vocabulaire - ou pour affirmer l'existence d'un sanctuaire sans autre précision ; (2) le hieron désigne un lieu sacré clairement dépourvu de naos, (3) ou, au contraire, un sanctuaire avec une structure bâtie, (4) soit, enfin, le naos lui-même. Certains de ces emplois pouvant se recouper, une telle distinction est surtout pragmatique et opératoire.

(I) Dans les énumérations de lieux sacrés, il est souvent difficile de préciser à quelles réalités topographiques ou architecturales correspond le hieron. En effet,

6 Le chiffre indiqué entre parenthèses correspond à l'ensemble des occurrences du mot qui précède.

7 La limite physique de l'enclos est parfois une "barrière de pierres " (Өрıүко̀ $\lambda \hat{i} \theta \omega v)$ : I, 42, 7 ; II, I5, 3 ; 35, IO ; V, I3, I ; VI, 20, 7 ; 25, I ; VIII, 3I, 5 ; 37, IO ; X, 38, 6 (2 occ.).

8675 occurrences, dont une restitution.

9 Casevitz, "Temples et sanctuaires ", loc. cit. (note 4) 82-85. - En Linéaire B, le neutre sub-

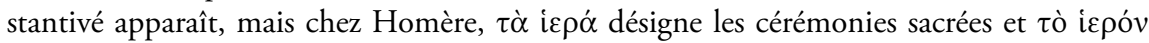
n'est pas employé (ibid. 82-83). Cf. aussi J. Rudhardt, Notions fondamentales de la pensée religieuse et actes constitutifs du culte dans la Grèce classique $\left(1958,1992^{2}\right)$ 23, 26-27.

IO Paus. I, 2, 5; 5, 5; I3, 8 ; 2I, 7 ; 29, 2 ; 29,3 ; II, 5, 5; 20, 9; 30, IO ; 34, IO ; III, 2I, 4 ; IV, 5, 9 ; 7, IO ; 23, 8 ; 27, 5-7 ; 29, IO ; 34, II ; V, 5, 6 ; 20, 5 ; VI, I8, 4 ; VII, 5, 4 ; I5, IO ; 25, 8 ; VIII, 2I, 3 ; IX, I, $8 ; 7,6 ; 22,2 ; 30, I I_{3} ; 37,5 ; \mathrm{X}, \mathrm{I} 9,8 ; 22,6 ; 33,4 ; 35,2$. 
son caractère général offre une belle opportunité de variatio sermonis dans une série de termes plus spécialisés. Ainsi, dans l'ancien port athénien du Phalère, se trouvent un hieron de Déméter, un naos d'Athéna Skiras, un autre de Zeus, des autels, ${ }^{\text {II }}$ sans qu'il soit possible de préciser quel type de sanctuaire était dédié à Déméter. De même - et l'on pourrait multiplier les exemples ${ }^{12}-$, au flanc sud de l'acropole athénienne se succèdent un hieron d'Asclépios, un naos de Thémis, un mnèma d'Hippolyte, des statues d'Aphrodite Pandemos et de Peitho, un hieron de Déméter Chloè et Gè Kourotrophos. ${ }^{13}$ Le sanctuaire d'Asclépios a été fouillé et comprenait plusieurs édifices construits, ${ }^{14}$ tandis que les statues d'Aphrodite et de Peithô désignent par synecdoque le petit temple qui les accueillait. ${ }^{\text {IS }}$ Le vocabulaire employé par Pausanias ne permet donc pas d'identifier l'apparence des éléments qu'il désigne de la sorte. De la même manière, au sommet de l'acropole Karia de Mégare se succèdent un naos de Dionysos Nyktelios, un hieron d'Aphrodite, un manteion de la Nuit, un naos de Zeus Konios, des agalmata d'Asclépios et Hygie réalisées par Bryaxis, un megaron de Déméter. ${ }^{16}$ L'archéologie n'est d'aucun secours et l'on ne peut guère déterminer à quoi ressemblait le hieron d'Aphrodite ou quelle structure accueillait les statues de Bryaxis, si c'était bien le cas. ${ }^{17}$

Une telle incertitude n'est cependant pas le seul fait d'un hieron s'inscrivant dans une série descriptive. On rencontre de nombreux cas où la mention isolée d'un hieron, en l'absence de tout indice complémentaire, ne permet pas de dépasser le simple constat de l'existence d'un "sanctuaire " en ce lieu. ${ }^{18}$

(2) Tout aussi nombreux sont les cas où l'on est incapable d'affirmer, sans autre élément pour asseoir une quelconque certitude, que hieron désigne un sanctuaire dépourvu de temple, à l'exception du hieron d'Apollon situé dans une grotte du

II Paus. I, I, 4 .

I2 E.g. Paus. II, 23, 2-4 ; 24, I-2 ; 27, 5 ; VII, 2I, IO-II ; 23, 9 ; 24, I ; X, 38, I2.

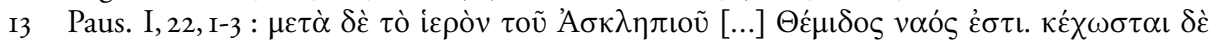

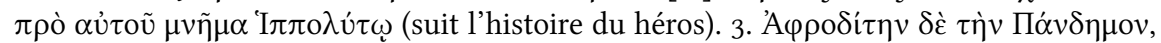

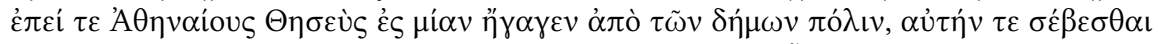

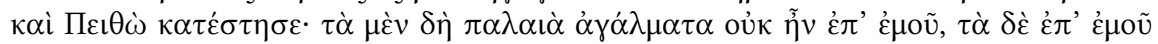

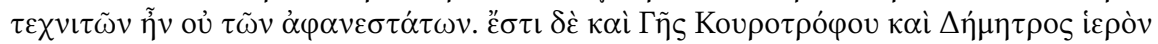
$\mathrm{X \lambda óns.}$

I4 Cf. J. W. Riethmüller, Asklepios. Heiligtümer und Kulte I (2005) 250-273.

I5 Cf. V. Pirenne-Delforge, L’Aphrodite grecque (1994) 26-34.

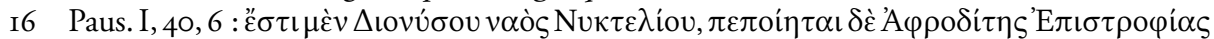

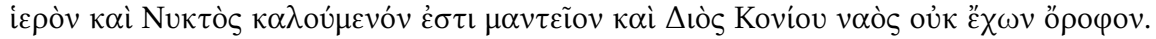

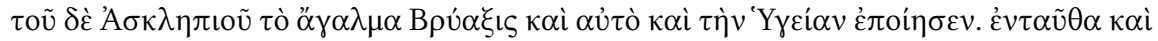

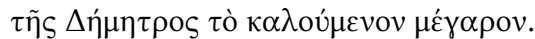

I7 A. Muller, "Megarika I-II », Bulletin de Correspondance Hellénique Io4 (1980) 83-92.

I8 E. g. le hieron de Peitho à Sicyone (II, 7, 7-8); celui d'Artémis Peitho à Argos (II, 2I, I); celui d'Aphrodite Nymphia entre Hermione et Trézène (II, 32,7); ceux de Poséidon et Artémis à Sparte (III, I4, 2); ceux d'Asclépios et Aphrodite à Cyllène (VI, 26, 5); ceux de Dionysos et Artémis à Phelloè (VII, 26, II) ; ceux de Déméter, Dionysos et Sarapis à Kopai (IX, 24, I). 
flanc sud de l'acropole d'Athènes, ${ }^{19}$ ou de celui des Praxidikai d'Haliarte que Pausanias localise en plein air. ${ }^{20} \mathrm{La}$ seule mention de statues ou d'autels dans un hieron n'implique pas nécessairement l'absence d'une structure bâtie de plus grande envergure, dans la mesure où les sélections opérées par le visiteur lui font souvent préférer la partie au tout, laissé dans l'ombre d'une appellation générique commode. ${ }^{2 \mathrm{I}}$

(3) Ce générique peut aussi s'appliquer à un ensemble consacré dont les composantes sont clairement décrites. La mention d'un hieron sert alors d'introduction à un panorama plus précis. Le sanctuaire de Dionysos au pied de l'acropole athénienne en offre un très bel exemple. Qualifiant tout d'abord le hieron de très ancien, Pausanias précise que deux naoi s'élèvent dans le peribolos, pour terminer par l'évocation des deux statues de culte et la description de peintures. ${ }^{22}$ De la même manière, le hieron d'Amphiaraos à Oropos accueillait un naos, une statue en marbre blanc et Pausanias décrit également l'autel compartimenté. ${ }^{23}$ De tels emplois sont heureusement nombreux ${ }^{24}$ et confirment le caractère général de l'appellation, que ce soit dans les parties descriptives de l'œuvre ou dans ses narrations. ${ }^{25}$

(4) Néanmoins, la signification de "temple ", et donc la synonymie avec naos, est bien attestée. La variatio sermonis dans la description d'un même sanctuaire peut justifier un tel usage. À Hermione, le hieron de Déméter Chthonia est remarquable, de même que la fête qui s'y tient tous les ans. Dans la description du mode de sacrifice en l'honneur de la déesse, Pausanias alterne l'utilisation de hieron et de naos pour désigner le temple de Déméter où les animaux pénètrent avant d'être

I9 Paus. I, 28, 4 : Ađ

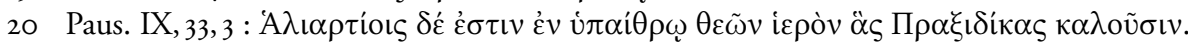

2I E.g. Paus. I, I7, 2; 19,3; 23,7 ; 28,6;33,2 (Némésis à Rhamnonte, où les fouilles ont mis au jour des restes de temple) ; I, 40, 2 ; II, 33, 3 ; III, 22, 8 ; VII, 23, 7 ; VIII, 37, II ; X, 34, 7 .

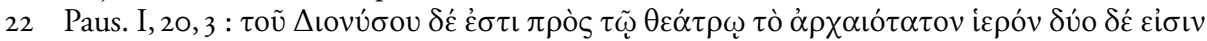

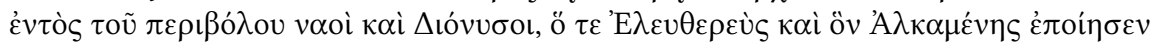

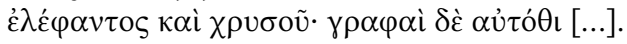

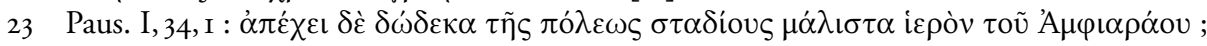

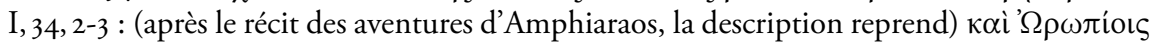

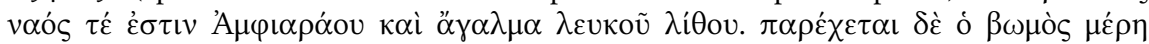
[...].

24 E. g. Paus. I, 8, 4 (Arès à Athènes) ; I, I8, 6 (Zeus Olympien à Athènes) ; II, I, 7 (Poséidon sur l'Isthme) ; Apollon Lykios à Argos (II, 19, 3-7) ; II, 26, I (Asclépios à Épidaure) ; III, I6, 6 (Lycurgue à Sparte) ; III, 26, I (Ino sur la route entre Oitylos et Thalamai) ; VI, 20, 2 (Ilithyie à Olympie); VIII, 37, I (Despoina à Lykosoura); X, 2, 2 (et tout au long du livre X, Apollon à Delphes).

25 E. g. Paus. I, 36, 4 par rapport à I, I, 4 pour l'Athéna du Phalère ; I, 4I, 3, naos d'Artémis et Apollon à Mégare, hieron pour conclure le mythe fondateur ; II, 5,6 par rapport à II, II, I pour l'Athéna de Sicyone; VI, 25, 2 par rapport à VI, 25, 3 pour l'Hadès d'Élis ; VII, I9, 3 par rapport à VII, 22, II pour l'Artémis Triklaria à Patras ; VIII, 4, 8 par rapport à VIII, 45, 4-5 pour l'Athéna Alea de Tégée. 
égorgés. ${ }^{26}$ Dans d'autres cas, plus nombreux, le détail de la description montre que Pausanias parle d'un naos sous le terme hieron: un toit, ${ }^{27}$ des portes, ${ }^{28}$ un plafond ${ }^{29}$ des colonnes, ${ }^{30}$ des briques ${ }^{31}$ sont autant d'indices clairs de cette synonymie. Un hieron en ruines semble de même n'être souvent qu'un naos ayant subi les outrages du temps ou des hommes. ${ }^{32}$ Un hieron dont on ne peut voir l'intérieur à la suite d'un interdit peut éventuellement être un naos, mais aussi un espace enclos d'un mur suffisamment haut pour entraver la vue de l'intérieur. ${ }^{33}$ Enfin, quand une des-

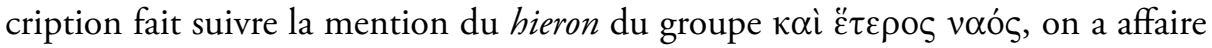
à deux temples. ${ }^{34}$

Il reste à envisager quelques passages complexes. Le premier apparaît au livre II, lors de la visite de Sicyone. À la faveur de la mention d'une statue d'Héraclès dans le gymnase de l'agora, Pausanias enchaîne en précisant que :

Ailleurs se trouve un hieron d'Héraclès; ils appellent Paidizè tout le peribolos à cet endroit, au milieu duquel se trouve le hieron avec un xoanon ancien à l'intérieur, une œuvre de Laphaès de Phlionte. ${ }^{35}$

Si tout le peribolos est bien consacré à Héraclès, ${ }^{36}$ le premier hieron désigne le sanctuaire au sens large, et le second, le temple dans lequel se trouve le xoanon. En revanche, si le Paidizè n'est pas comme tel un "sanctuaire d'Héraclès ", les deux mentions de hieron désignent le lieu consacré à l'intérieur d'une enceinte "profane " et rien ne permet de l'identifier à un temple. La première hypothèse semble la plus probable, comme le montre la suite immédiate de la visite de Pausanias. Une route le conduit, en effet, vers le hieron d'Asclépios :

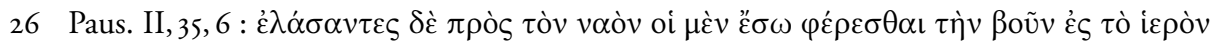

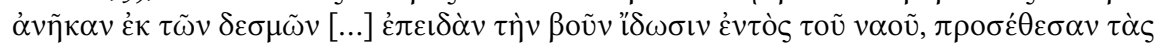
$\theta u ́ p \alpha \varsigma$. - Bon nombre d'occurrences en alternance avec naos pour un même sanctuaire ne permettent cependant pas d'être aussi affirmatif : hieron est soit le générique qui désigne le sanctuaire intégrant le temple, soit le temple lui-même ; e. g. II, 2I, 8-IO et 22, I ; III, I7, 2 ; VIII, 25, 4 \& 6 ; 3I, 5-6; IX, 33, 5-6.

27 Paus. I, 44, 3 ; II, 34, IO ; III, 22, IO.

28 Paus. II, 2I, 4.

29 Paus. III, I6, I.

30 Paus. VIII, 44, 2.

3 I Paus. V, 5, 6; IX, 19, 5; 25, 3 ; X, 36, 8. Cf. IX, 16, 6.

32 Paus. II, 9, 7; 36, 8 ; VI, 20, 6; 2I, 3; VI, 2I, 6 ; VIII, I2, 9; 24, 6; 25, 3 ; 3I, 9; 32, 2 (mention

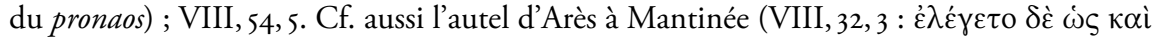

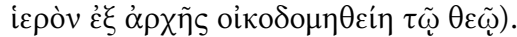

33 Paus. III, 20, 8 ; VIII, 4I, 4 ; IX, I9, 5 ; 25, 3. Cf. IX, I6, 6 et X, 35, 7, où le naos apparaît comme tel.

34 E.g. Paus. II, 25, 6.

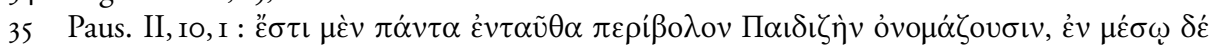

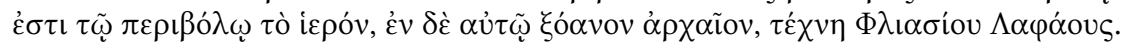

36 Cf. infra, pour l'étude du mot. 
En entrant dans le peribolos se trouve sur la gauche un bâtiment double [...] à l'entrée de l'Asclépieion s'élèvent de part et d'autre de l'entrée une statue de Pan assis et une Artémis debout ; à l'intérieur se trouve le dieu, imberbe, fait d'or et d'ivoire, une œuvre de Kalamis. ${ }^{37}$

Le hieron introductif qualifie le peribolos dans sa totalité et la mention de l'Asclépieion fait surgir le temple dans la description. Le vocabulaire du paragraphe suivant est similaire : le peribolos voisin est consacré à Aphrodite, enferme une statue d'Antiope et, enfin, le hieron d'Aphrodite. Ce hieron est sans conteste le temple, puisque tout le peribolos est consacré à la déesse. De surcroît, Pausanias précise que les fidèles doivent prier la déesse en regardant la statue "depuis l'entrée ». Comme la statue était chryséléphantine et devait donc être protégée, l'entrée en question doit être celle du naos. En outre, une plante qui intervient dans le sacrifice pour la déesse ne pousse nulle part ailleurs que dans la partie à l'air libre du peribolos $: 3^{38}$ cela implique l'existence de constructions en son sein.

Toujours au livre II, sur la route de Mycènes à Argos, se trouve un hieron de Déméter Mysia, dont l'épiclèse rappelle l'hospitalité que lui accorda jadis l'Argien Mysios. Le toit a disparu, "et à l'intérieur se trouve un autre naos en briques cuites $»{ }^{39}$ Le hieron est donc bien un naos endommagé à l'intérieur duquel un nouveau temple a vu le jour. Dans le même ordre d'idée, une structure célèbre se situe sur la route de Mantinée à Tégée, en Arcadie. Pausanias y évoque le hieron de Poséidon Hippios frappé d'un lourd interdit. Il s'agit d'une construction de l'empereur Hadrien qui s'est faite sous " haute sécurité ", dans la mesure où aucun ouvrier n'avait le droit de jeter un œil sur les débris de l'ancien hieron ni d'en déplacer le moindre reste. Dès lors, le nouveau naos a été bâti tout autour des ruines dont l'origine remontait à la structure de poutres en chêne ajustée par Agamédès et Trophonios..$^{40}$ Chaque occurrence du mot hieron renvoie à un naos, l'ancien et le nouveau qui l'englobe.

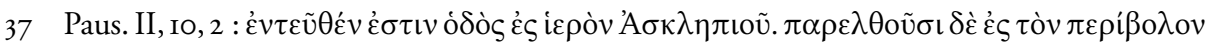

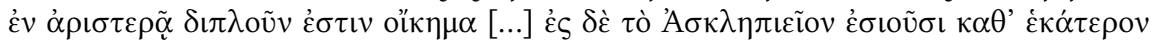

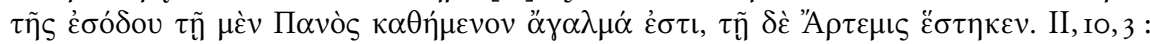

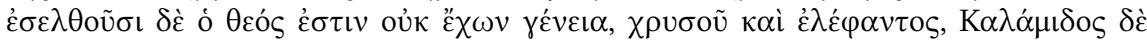
हैंpүov.

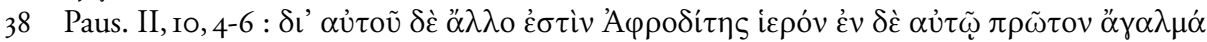

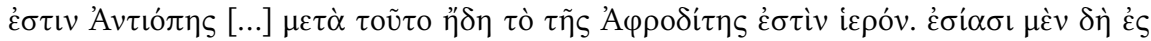

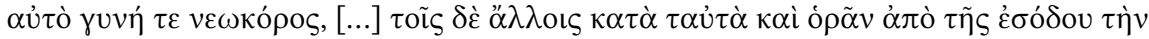

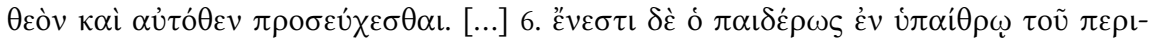

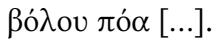

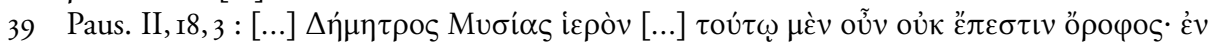

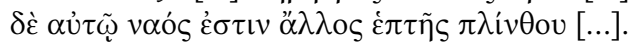

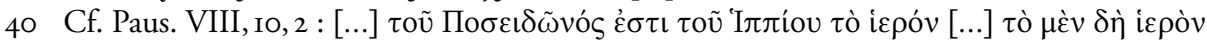

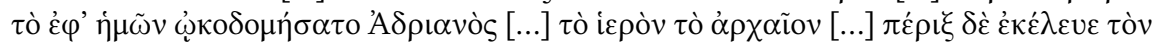

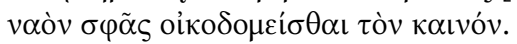


La grande majorité des emplois de hieron, quand le mot est déterminé par le nom de son propriétaire, concerne des divinités, locales ou olympiennes. À quelques reprises, cependant, Pausanias parle du hieron d'un personnage que nous qualifions d'héroïque ${ }^{41}$ et pour lequel on s'attendrait davantage à la mention d'un hèrôon. Ce dernier mot sera envisagé plus loin, ${ }^{42}$ mais tentons dès à présent de comprendre le choix de la désignation en hieron. Une telle attribution peut s'expliquer par l'interprétation divine du personnage à l'échelon du culte local. ${ }^{43}$ C'est le cas pour le hieron que les Spartiates ont élevé au législateur Lycurgue " comme à un dieu ${ }^{44}$ et aux différents héros guérisseurs apparentés à Asclépios..$^{45}$ Quant à Baton, le cocher d'Amphiaraos, peut-être son statut est-il assimilé à celui de son maitre : tous deux ont été aspirés par la terre entrouverte. ${ }^{46}$ Dans ce cas précis, l'absence de tombeau est un critère important. C'est probablement le cas aussi pour le hieron de Kychreus, à Salamine, que l'oracle de Delphes avait identifié sous les traits d'un serpent apparu pendant la bataille de Salamine. ${ }^{47}$ Son lieu de culte sur l'île était indépendant de tout tombeau.

Le cas de Thésée à Athènes montre toutefois qu'une telle explication ne peut être appliquée de façon mécanique. Pausanias consacre cinq paragraphes au récit des exploits du héros à la faveur de la description de son sanctuaire de l'agora. ${ }^{8}$ Il s'agit d'un hieron accueillant les représentations peintes de ses hauts faits. C'est

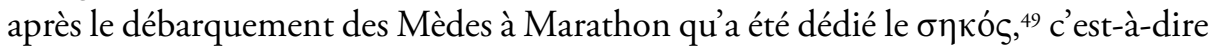
l'enceinte pour Thésée. C’est la seule occurrence de sèkos dans toute la Périégèse.

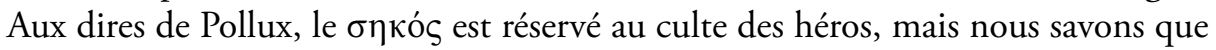

4I Thésée à Athènes (I, I7, 2) ; le héros Kychreus à Salamine, sorte de démon ophidien (I, 36, I), Métanire près d'Éleusis (I,39,2), les Leucippides (III, I2, 8; I6, I), Achille (III, 20,8) et Hélène (III, I5,3) à Sparte, Dryops à Asinè (IV, 34, II). Cf. aussi notes suivantes. - Indépendamment d'une description directe est encore mentionné le hieron d'Hélène à Rhodes (III, I9, IO).

42 Cf. infra, p. 163-165.

43 Sur les variations rituelles dans le culte des « héros », voir notamment G. Ekroth, The Sacrificial Rituals of Greek Hero-Cults (2002).

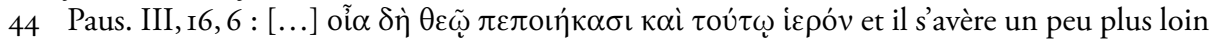

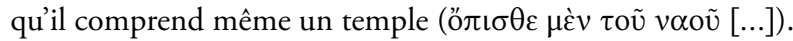

45 Machaon (III, 26, 9), ses enfants (IV, 3, 2), Polémokratos (II, 38, 6). Peut-être Mélampous à Aigosthènes entre-t-il dans cette catégorie (I, 44, 5).

46 Paus. II, 23, 2.

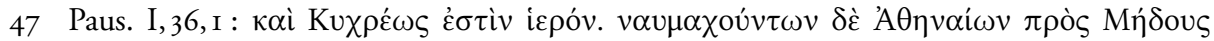

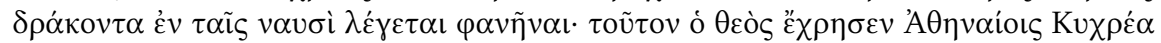

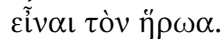

48 Paus. I, I7, 2-6.

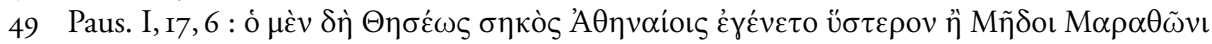

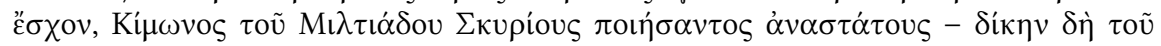

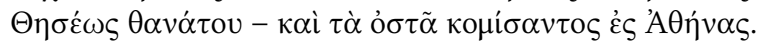


les poètes parlent aussi de sèkos des dieux..$^{\circ}$ Le Theseion remontant au moins au milieu du $\mathrm{VI}^{\mathrm{e}}$ siècle, ${ }^{\mathrm{II}}$ le sèkos est peut-être l'enclos aménagé à l'intérieur du hieron lors du rapatriement des ossements du héros par Cimon peu après 476.52 Quoi qu'il en soit, ce passage est manifestement inspiré de la Vie de Cimon de Plutarque qui parle lui aussi d'un sèkos pour Thésée..$^{53}$

Le hieron est donc le lieu sacré par excellence et son attribution à une personnalité divine est largement prédominante, même si le terme n'est pas incompatible avec un propriétaire « héroïque ». Son emploi par Pausanias rencontre à la fois le souci de varier son style et de rendre compte des realia du terrain. Toutefois, cette dernière préoccupation est parfois fortement compromise par la première. Il ne faut pas en outre exclure la volonté, affichée par l'auteur, de négliger des informations jugées inutiles. Bon nombre d'occurrences conduisent dès lors au simple constat de l'existence d'un sanctuaire dont l'ordonnancement reste obscur.

\section{2. $\mathrm{N} \alpha o ́^{54}$}

Le mot désigne toujours un édifice construit, qu'il forme le sanctuaire à lui seul ou qu'il en soit la réalisation architecturale maîtresse. ${ }^{5}$ Pausanias ne prend que rarement la peine de décrire le naos avec précision, mais deux passages permettent de

50 Pollux I, 6. - Une inscription de Lébadée (Inscriptiones Graecae VII 3077,1.2) datée de la fin du $\mathrm{I}^{\mathrm{er}} \mathrm{s}$. - début $\mathrm{II}^{\mathrm{e}} \mathrm{s}$. ap. J.-C. évoque les réparations à effectuer au péribole (? le mot est restitué) du sèkos sacré de Zeus Trophonios. Il devait s'agir de la grille qui entourait l'entrée de l'adyton, dont l'appellation locale fait donc un sèkos. Cf. M.-Chr. Hellmann, Choix d'inscriptions architecturales grecques traduites et commentées (1999) n I3. Pausanias n'utilise pas le terme de sèkos à Lébadée (cf. infra, n. 187).

5I En relation avec la prise de pouvoir de Pisistrate : Aristote, Athenaion Politeia 15, 4. Cf. aussi Thucidide VI, 6I ; Andocide, Sur les mystères 45.

52 Le point sur la question chez Cl. Calame, Thésée et l'imaginaire athénien (1990) I54 et n. 32, I8O-I8I.

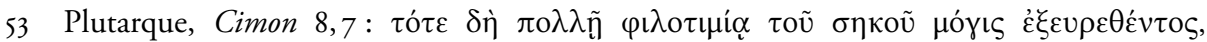

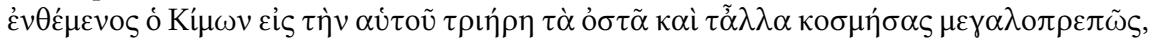

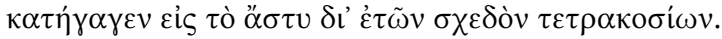

54446 occurrences dont 3 citations.

55 C'est la raison pour laquelle il n'est guère évident, en se fondant sur son témoignage, de considérer que le naos d'Athéna Polias qu'il situe sur l'acropole d'Athènes est inclus dans l'Érechtheion $(\mathrm{I}, 27,2)$. Je reprends ce dossier dans une étude spécifique à paraître dans les Mélanges en l'honneur de Madeleine Jost. La discussion a été récemment ranimée par des études fouillées : e.g. M. Osanna, "Pausania sull'Acropoli: tra l'Atene di Endoios e l'agalma caduto dal cielo ", Mélanges de l'École Française de Rome, Antiquité II3 (200I) 32I- 
dessiner le noyau minimal de la structure qu'il désigne sous ce terme. Tout d'abord à Sicyone, évoquant une forme particulière de sépulture, il explique que les gens du lieu « ensevelissent le corps en pleine terre et construisent par dessus un soubassement de pierre qui porte des colonnes surmontées d'un couronnement, reprodui-

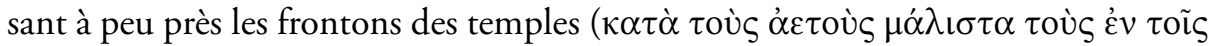

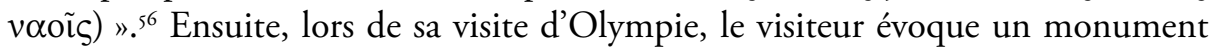
funéraire " en forme de naos : il n'est pas très haut, il n'a pas de mur et des colonnes de chêne soutiennent le toit $" .{ }^{57}$ Pausanias ne fera pas le lien entre les traditions sicyoniennes et ce tombeau anonyme, mais le rapprochement de ces deux passages livre la structure architecturale minimale qui commande l'usage du terme de naos: un soubassement, des colonnes et des frontons. Mais, une fois cette architecture repérée - fût-ce avec toutes les variations qu'atteste pour nous l'architecture religieuse, ${ }^{58}$ - encore faut-il que le propriétaire du lieu soit une divinité : colonnes et frontons de propylées, par exemple, ne pourront évidemment suffire.

Les évocations de naoi restent souvent désespérément allusives. On apprend parfois que la construction n'est pas grande ${ }^{59}$ ou, au contraire, que sa taille est exceptionnelle, ${ }^{60}$ que le temple est en briques, ${ }^{61}$ en marbre, ${ }^{62}$ ou même en pierre locale. ${ }^{63}$ Il lui arrive de préciser qu'il est inachevé, ${ }^{64}$ endommagé, ${ }^{65}$ ruiné, ${ }^{66}$ qu'il a été foudroyé ${ }^{67}$ ou simplement qu'il est ancien. ${ }^{68}$ Un aménagement particulier reçoit aussi une attention plus précise : à Sparte, un des sanctuaires d'Aphrodite est un

340 ; G. Ferrari, "The Ancient Temple on the Acropolis at Athens ", American Journal of Archaeology IO6 (2002) II-35; J. Pakkanen, "The Erechtheion Construction Work Inventory (IG I ${ }^{3} 474$ ) and the Dörpfeld Temple ", American Journal of Archaeology IIo (2006) 275-28I ; H. Gerding, "The Erechtheion and the Panathenaic procession ", American Journal of Archaeology IIO (2006) 389-40I (avec une bibliographie complémentaire).

56 Paus. II, 7, 2 (trad. G. Roux, infra n. 83, p. 54).

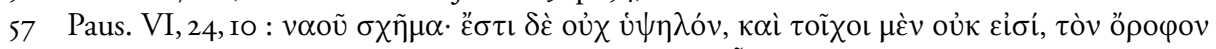

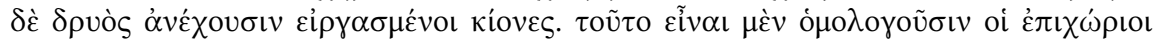
$\mu \nu \tilde{\eta} \mu \alpha$, ö $\tau$ ov $\delta \dot{\varepsilon}$ ov̉ $\mu \nu \eta \mu o v \varepsilon v ́ o v \sigma ı v$.

58 Sur ce point, la synthèse de M. Chr. Hellmann est particulièrement éclairante : L'architecture grecque. 2. Architecture religieuse et funéraire (2006) 28-34.

59 Paus. I, 29, 2 ; V, 20, 9 ; IX, 27, $5 ; \mathrm{X}, 35,4$.

60 Paus. IV, 3I, 8 ; VIII, 45, 4-5; IX, 2, 7 ; X, 35, 4.

6I Paus. I, 42,$5 ; \mathrm{II}, \mathrm{I} 8,3$.

62 Paus. I, 42, 5; VIII, 28, I ; 4I, 7.

63 Paus. VI, 27, 2.

64 Paus. IX, 4,$4 ; 39,4$.

65 Paus. I, I, 5; 40,6; II, 7,6;5,5; 7,9; II, $2 ; \mathrm{I2}, 2 ; 18,3 ; 24,3 ; 34,10 ; 36,2 ; \mathrm{III}, 2 \mathrm{I}, 8$; VI, 24, IO ; VIII, 4I, IO ; 44, 3 ; IX, 33, 3 et 7 ; X, 35, 2.

66 Paus. VIII, 9, 6 ; I4, 4 ; I5, 5; 26, 2 ; 30, 6 ; 3I, 9; 32, 3; 36, 8; 53, II ; X, 8, 6.

67 Paus. II, II, I ; VIII, 32, 3.

68 Paus. VIII, 22, 7 . 
temple à étage, ce que Pausanias affirme n'avoir jamais vu ailleurs $;^{69}$ toujours en Laconie, au cap Ténare, s'élève un naos en forme de grotte, précédé d'une statue de Poséidon $;^{70}$ à Mantinée, Léto et ses enfants partagent avec Asclépios un naos double divisé par un mur. ${ }^{71}$

Même quand l'importance du culte ou la beauté du temple le conduit à s'arrêter plus longuement sur l'apparence d'un édifice, Pausanias, fidèle en cela aux principes généraux de son ouvrage, sélectionne l'information. Le Parthénon se voit ainsi gratifié de la seule évocation du thème de ses frontons : la naissance d'Athéna et la querelle entre la déesse et Poséidon pour la possession de l'Attique. ${ }^{72}$ L'Héraion argien se résume à quelques-unes de ses décorations sculptées: la naissance de Zeus, la gigantomachie, la guerre de Troie. ${ }^{73}$

La description du temple de Zeus à Olympie est davantage détaillée : l'édifice est dorique, périptère, en calcaire local ; on en connait les mesures; ses tuiles sont en marbre pentélique et les décorations sculptées extérieures sont passées en revue. Véritable écrin pour la gigantesque statue du dieu, l'intérieur du temple est brièvement évoqué et la statue décrite..$^{74}$ Également situé dans l'Altis, l'Héraion, dont Pausanias précise aussi la taille, est dorique, périptère et l'une des colonnes de l'opisthodome est en chêne. ${ }^{75} \mathrm{La}$ description du naos proprement dit s'arrêtera là.

Selon Pausanias, de tous ceux du Péloponnèse, le temple d'Apollon à Bassai est le premier, après celui de Tégée, par la beauté de ses pierres et l'harmonie de ses proportions ${ }^{76}$ mais on n'en saura pas plus. Quant à ce temple d'Athéna Alea à Tégée, dont - Pausanias l'affirme une fois encore - la structure et la taille sont exceptionnelles, il apparaît plus précisément dans une description attentive : la première rangée de colonnes est dorique et la suivante, corinthienne ; à l'intérieur, l'ordre est ionique. Les frontons sont également décrits : chasse de Kalydon d'un côté, combat de Télèphe et d'Achille de l'autre. ${ }^{77}$

Comme pour le hieron, les propriétaires de ces naoi énumérés par le visiteur sont essentiellement des divinités, locales ou olympiennes. Les quelques " héros " qui s'en voient attribuer en certains endroits assument à l'échelon local un statut

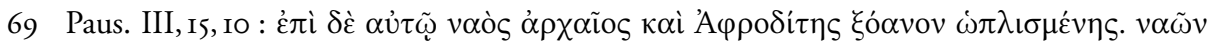

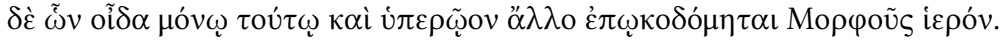

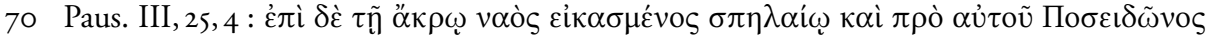
$\ddot{\alpha} \gamma \alpha \lambda \mu \alpha$.

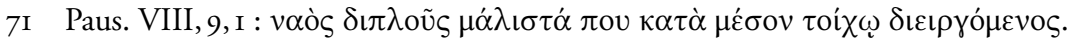

72 Paus. I, 24, 5 .

73 Paus. II, 17,3 .

74 Paus. V, IO, 2-IO.

75 Paus. V, I6, I.

76 Paus. VIII, 4I, 8.

77 Paus. VIII, 45, 5-7. 
différent. C'est le cas, notamment, d'Ajax à Salamine, ${ }^{78}$ d'Hippolyte à Trézène, ${ }^{79}$ d'Hélène et Ménélas à Thérapnè ${ }^{80}$ ou de Cassandre à Amyclées. ${ }^{81}$

\section{3. ${ }^{\prime} \mathrm{A} \lambda \sigma o \varsigma^{82}$}

Pausanias donne le nom d'alsos à un lieu boisé $e^{83}$ auquel la présence d'un ou de plusieurs sanctuaires confère sa sacralité. ${ }^{84}$ Grand amateur d'arbres particuliers, ${ }^{85}$ il précise à plusieurs reprises l'essence ou les essences de ceux qui poussent dans l'alsos, mais aussi le fait que les arbres soient tous ou en partie plantés de main d'homme, tous de la même essence ou non. ${ }^{86} \mathrm{Il}$ procède de la même manière dans

78 Paus. I, 35, 3 .

79 Paus. II, 32, I.

80 Paus. III, I9, 2.

8I Paus. III, 26, 5.

82 Sur 85 occurrences, 67 emplois s'inscrivent dans une description et concernent 44 endroits différents, I7 fois le mot est employé hors de toute description pour évoquer II lieux différents, et il apparaît également dans une citation.

83 À deux reprises, sans préciser l'espèce des arbres, il détermine $\alpha \chi \lambda \sigma o \varsigma$ par $\delta \varepsilon \dot{v} \delta \rho \omega \omega \nu$ (VIII, 35,6 ; VIII, 38, 5). Il serait absurde d'en déduire que l'alsos indéterminé n'était pas nécessairement boisé. Cf. les emplois repris chez Casevitz, "Temples et anctuaires ", loc. cit. (note 4) $9 \mathrm{I}-93$.

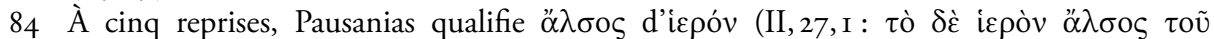
'A $\sigma \kappa \lambda \eta \pi เ o \tilde{v}$ à Épidaure ; II, 36, 8 : l'alsos de Lerne où Déméter et Dionysos étaient parti-

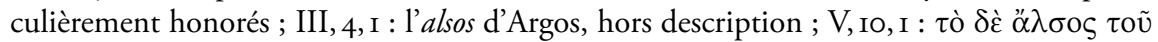

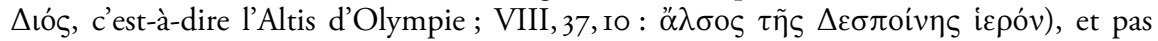
en II, II, 3 comme l'affirme Chr. Jacob, "Paysage et bois sacrés : " $\alpha \sigma o \varsigma$ dans la Périégèse de la Grèce de Pausanias ", in : O. de Cazenove - J. Scheid (éds.), Les Bois sacrés. Actes du Colloque International organisé par le Centre Jean Bérard et l'École Pratique des Hautes Études (Vection) (1993) 3I-44, spéc. 34. Pour une tentative de définition de l'alsos, voir P. Bonnechere, "The Place of the sacred grove (alsos) in the mantic rituals of Greece : the example of the oracle of Trophonios at Lebadeia (Boeotia) ", in : M. Conan (éd.), Sacred Gardens and Landscapes : Ritual and Agency (2007) I7-4I, spéc. 4I.

85 G. Roux, Pausanias en Corinthie (Livre II, I à I5) (I958) 96, a bien mis cette prédilection en évidence. Cf. plus récemment D. Birge, "Trees in the Landscape of Pausanias' Periegesis", in : S. Alcock - R. Osborne (éds.), Placing the Gods. Sanctuaries and Sacred Space in Ancient Greece (1994) 23I-245 et A. Jacquemin, "Les curiosités naturelles chez Pausanias", in : G. Siebert (éd.), Nature et paysage dans la pensée et l'environnement des civilisations antiques. Actes du Colloque de Strasbourg II-I2 juin 1992 (I996) I2I-I28.

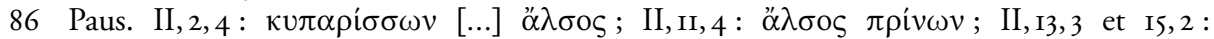

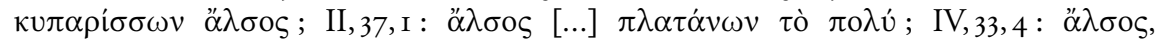

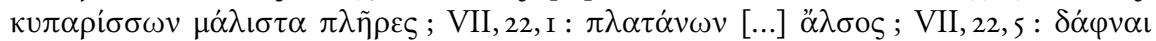

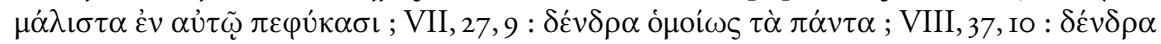

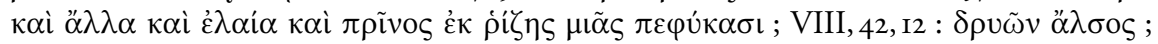

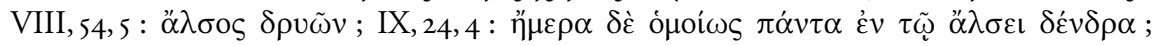


l'évocation de ses visites en Asie Mineure. ${ }^{87}$ Un aménagement particulier du site ou la présence d'une source d'eau vive peuvent aussi être signalés. ${ }^{88}$ À une exception près ${ }^{89}$ toutes les occurrences d'alsos dans la Périégèse présentent un rapport topographique immédiat, direct avec un ou plusieurs lieux de culte. Toutefois le rapport aux sanctuaires varie, sans qu'il soit jamais abusif de concevoir l'alsos comme un «bois sacré».

Parfois baptisé d'un nom particulier - Kraneion, ${ }^{90}$ Pyraia,${ }^{91}$ Karnasion ${ }^{92}-$, l'alsos peut, (I) soit accueillir différents sanctuaires, (2) soit être explicitement consacré à une divinité particulière ou à plusieurs dont le lien est affirmé, (3) soit renfermer un élément " religieux " sans que la relation entre les deux puisse être clairement établie, (4) soit abriter un sanctuaire de plus grande envergure, attribué de façon générique à un dieu, mais pouvant en accueillir d'autres, ou, enfin, (5) être intégré dans un sanctuaire sans se confondre avec lui.

(I) Dans le premier cas, ${ }^{93}$ la sacralité de l'alsos n'est pas explicitement affirmée dans un rapport privilégié à un seul culte. Le bois est alors conçu comme un lieu sacré, tout comme l'acropole d'une cité, dans sa concentration religieuse, peut être ainsi envisagée.

(2) Dans le deuxième cas, les expressions les plus claires attribuent comme déterminant à alsos le nom du dieu au génitif. ${ }^{94}$ Quand le déterminant divin pré-

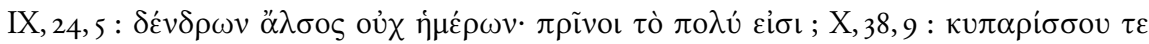

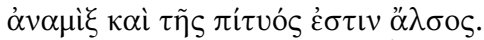

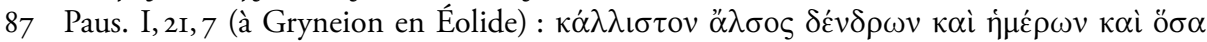

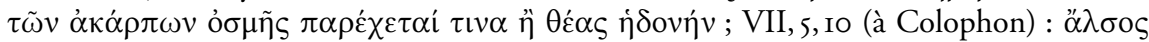

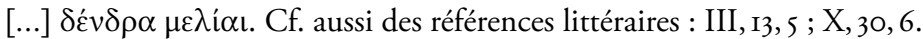

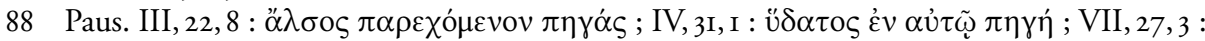

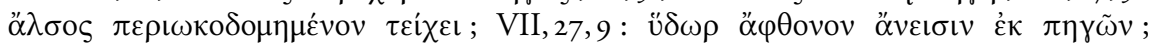

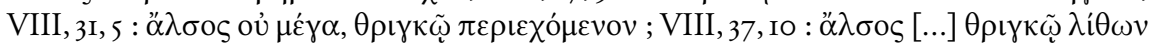

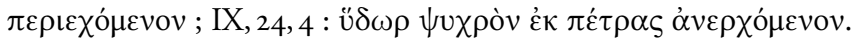

89 Sur la route vers Pharai, en Achaïe, Pausanias a vu un alsos de platanes que le temps a creusés et tellement grands que les gens pique-niquent ou dorment dans leurs troncs (VII, 22, I). - L'analyse qui suit prend uniquement en compte les 42 bois sacrés inclus dans une description topographique, et non les évocations de mémoire ou littéraires.

90 Paus. II, 2, 4.

9I Paus. II, II, 3 .

92 Paus. IV, 33, 4.

93 Paus. II, 2, 4 : dans l'alsos appelé Kraneion, à l'extérieur de Corinthe, se trouvent un temenos de Bellérophon, un naos d'Aphrodite et un taphos de Laïs ; VII, 2I, II : au rivage de Patras, en Achaïe, un alsos aux promenades rafraîchissantes en été abrite des temples de dieux, en l'occurrence Apollon et Aphrodite.

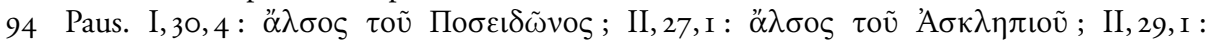

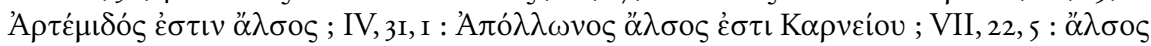

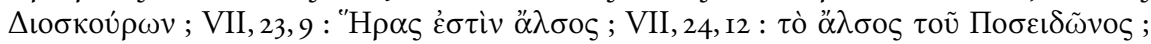

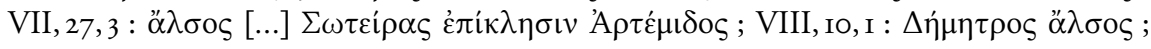




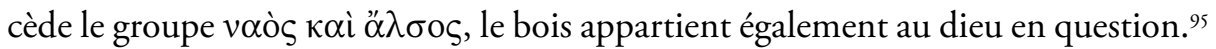
Parfois, des explications complémentaires viennent affirmer ce rapport. Ainsi sur

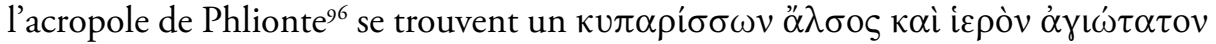
$\dot{\varepsilon} \kappa \pi \alpha \lambda \alpha$ เoṽ dont la propriétaire est Ganyméda, variante locale de Hèbè. ${ }^{97}$ Bienveillante pour les fuyards suppliants, la déesse reçoit l'hommage des anciens prisonniers qui suspendent aux arbres de l'alsos les liens qu'ils portaient aux pieds. L'alsos est incontestablement consacré à Hèbè puisqu'il accueille les marques d'action de grâce qui lui sont destinées. Quant au "sanctuaire très saint de longue date ", il semble renvoyer aux structures qui aménagent le lieu sacré de Ganyméda sur l'acropole de Phlionte, que ce soit dans l'alsos ou à côté de lui. Il n'est toutefois pas assuré que, dans ce cas précis, hieron soit le terme non marqué pour désigner un naos. En effet, à la fin de l'évocation du lieu et de sa déesse, Pausanias précise qu'il n'y a pas de statue, ni dans un endroit caché ni à la vue de tous, et qu'une telle absence fait localement l'objet d'un hieros logos. ${ }^{98}$ Une statue peut s'élever à l'air libre, mais a fortiori, lorsque le hieron qui s'ajoute à l'alsos est dépourvu de statue, il est d'autant moins certain que ce hieron soit un naos proprement dit.

Sur la route de Titanè, Pausanias a vu l'alsos de pins et le naos des déesses que les Sicyoniens appellent Euménides. Après avoir décrit le rite sacrificiel particulier qu'accueille leur autel, il conclut sur l'identité du rituel réservé aux Moires $\dot{\varepsilon} v$

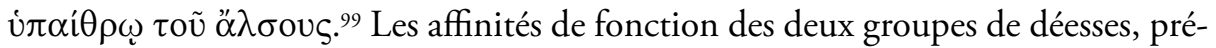
sentes dans les Euménides d'Eschyle, ${ }^{100}$ permettent d'affirmer la cohérence cultuelle du bois sacré et son statut de sanctuaire conjoint des Euménides et des Moires. À Lerne, la configuration du lieu est plus complexe. L'alsos est vaste et Pausanias en indique les limites : le mont Pontinos, la mer, les fleuves Amymoné et Pontinos. ${ }^{\text {Ior }}$ On y trouve des agalmata de Déméter Prosymné, de Dionysos, et un petit agalma

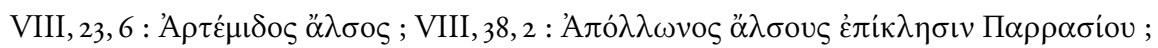

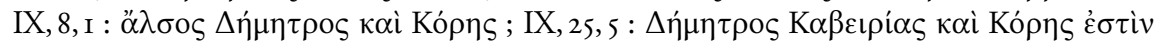

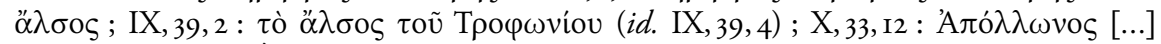

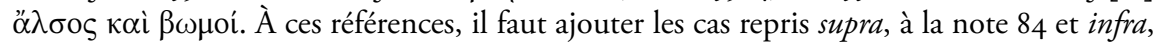
n. 95 .

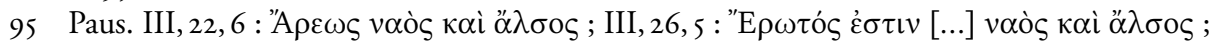

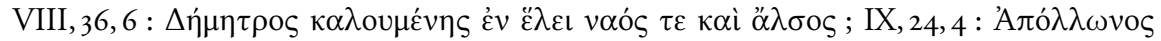

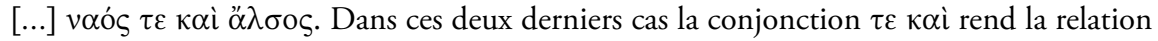
entre le naos et l'alsos tout à fait claire.

96 Paus. II, 13,3-4.

97 Si l'on en croit Strabon, Dia est le nom local d'Hèbè (VIII, 6, 24). Il use du terme hieron pour évoquer son sanctuaire.

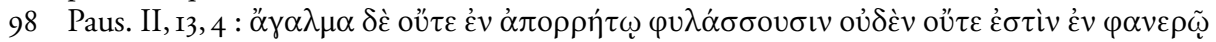

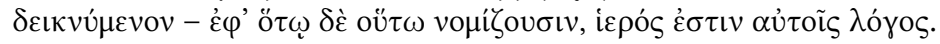

99 Paus. II, II, 4.

IOo Eschyle, Euménides 956-967.

IOI Paus. II, 37, I. 
assis de Déméter. Ailleurs, dans un temple, il y a un xoanon assis de Dionysos Saôtès et un agalma d'Aphrodite "sur la mer » en pierre..$^{102}$ Pausanias évoque, peu après les mystères de Lerne, une célébration que patronne Déméter et tenue dans l'alsos, de même qu'un rituel secret pour Dionysos. ${ }^{103}$ Cet alsos a une tonalité mystérique qui pourrait expliquer pourquoi Pausanias le qualifie de hieron juste avant d'en entreprendre la description. ${ }^{\mathrm{I} 4}$

Les occurrences d'alsos au livre $\mathrm{X}$ méritent un traitement à part. Elles sont au nombre de quatre, relevant directement d'une description. À Tithoréa, cité en déclin à l'époque, Pausanias décrit, en fait, de curiosités remarquables, A $A \eta v \tilde{\alpha} \varsigma$

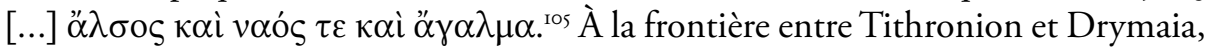

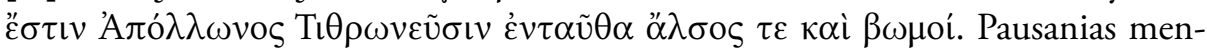

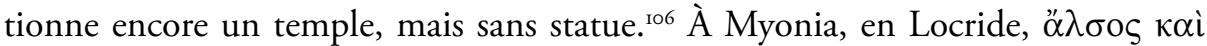

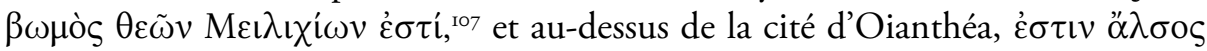

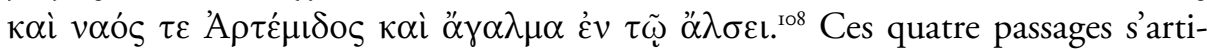
culent sur un même schéma de juxtaposition des éléments qu'intègrent les "bois sacrés " de Phocide et de Locride. La pauvreté des environs de Delphes, que Pausanias parcourt pourtant, explique sans doute une description plus " mécanique » qu'en d'autres lieux foisonnants de ces curiosités dignes de mémoire.

(3) Pausanias signale parfois l'existence d'un alsos autour d'un hieron, d'un naos, d'un bômos, ${ }^{109}$ d'un agalma. Dans bon nombre d'endroits, le bois faisait sans doute partie intégrante du sanctuaire, mais les soucis stylistiques de l'auteur ne permettent pas toujours de l'affirmer avec certitude. Le cas de Némée est exemplaire. Le visiteur décrit le naos de Zeus qui vaut la peine d'être vu, même si le toit s'est

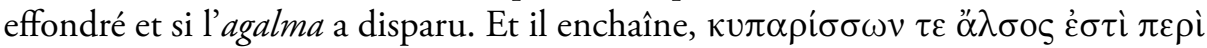

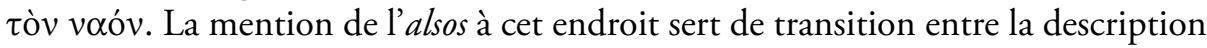
du lieu et l'histoire d'Opheltès, déposé sur l'herbe par sa nourrice et mordu par un serpent. ${ }^{\text {IIO }}$ L'alsos faisait probablement partie intégrante du sanctuaire, mais ne se confondait pas nécessairement avec la totalité du site. En revanche, dans les cas où

IO2 Paus. II, 37, 2.

IO3 Paus. II, 37, 2-6.

I04 Paus. II, $36,8$.

I05 Paus. X, 32, Io.

I06 Paus. $\mathrm{X}, 33$, I2.

I07 Paus. X, $38,8$.

I08 Paus. $\mathrm{X}, 38$, 9. Il précise que les murs étaient recouverts de peintures, mais que les effets du temps les ont effacées.

I09 Le cas des Dioscures des environs de Pharai en Achaïe est clair : l'alsos leur appartient et on n'y trouve plus ni temple, ni statues, mais bien un autel ; Paus. VII, 22, 5 .

IIO Paus. II, I5, 2 et 9. 
l'alsos est dit se situer $\pi \varepsilon \rho \grave{~} \tau$ tò ípó $\nu,{ }^{\text {III }}$ il est probable que le sanctuaire, qu'il soit ou non affecté d'un naos, se confondait avec l'alsos.

La formulation de l'ordonnancement peut également s'inverser : le hieron est alors situé à l'intérieur d'un alsos. Ainsi, sur la route entre Sicyone et Phlionte s'élève

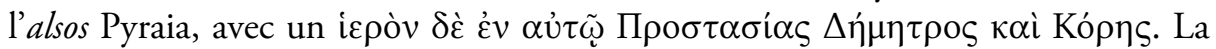
structuration des lieux devait être complexe car Pausanias précise que les hommes y célèbrent une fête entre eux, laissant aux femmes le Nymphôn qui abrite des statues de Dionysos, de Déméter et de Korè dont seuls les visages sont apparents. ${ }^{112} \mathrm{Le}$ Nymphôn est vraisemblablement un bâtiment laissé à la disposition des femmes. Quant à la fête des hommes, si elle n'était pas frappée d'un quelconque interdit, elle pouvait se tenir à l'air libre. Le statut de l'alsos Pyraia semble intimement lié au culte de Déméter et de sa fille, mais leur hieron pouvait être une zone plus réduite à l'intérieur du bois.

Entre Tégée et Argos, un alsos de chênes abrite un naos de Déméter c̉v

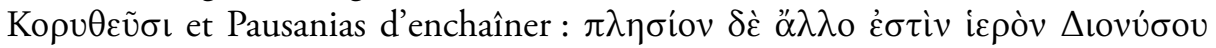

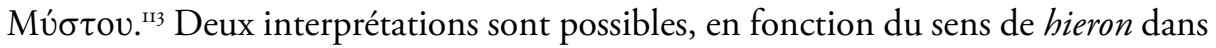
cette phrase. Soit hieron est synonyme de naos et le temple de Dionysos se trouve dans l'alsos en tant qu' " autre " temple, soit hieron est utilisé comme générique, ce qui fait du lieu de culte de Dionysos le pendant sacré de l'alsos de Déméter entendu comme "sanctuaire ». Dans ce cas précis, la qualité d' «initié » de Dionysos instaure un lien privilégié entre son culte et celui de Déméter. Il est donc probable que la première interprétation est la bonne. ${ }^{\mathrm{II}}$

Pausanias ne fait parfois référence qu'à un autel ou à une statue présents dans un alsos. Ainsi, à un demi-stade de Korseia, en Béotie, se trouve un alsos de pins

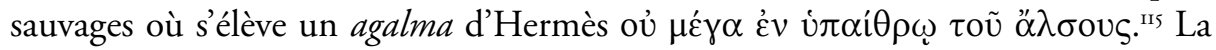
statue est manifestement indépendante de toute construction et rien ne permet de préciser les relations existant entre l'alsos et Hermès.

(4) Le quatrième cas de figure fait d'un alsos de grande taille la structure d'accueil d'un lieu de culte important auquel sont subordonnés toute une série de divinités ou de héros. Les deux exemples les plus célèbres sont le sanctuaire d'Asclépios

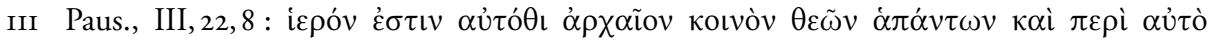

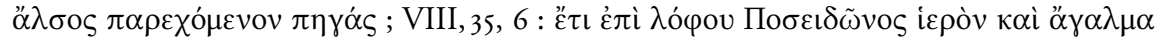

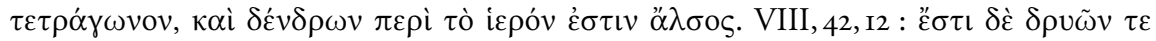

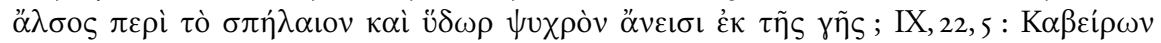

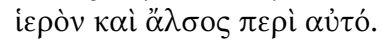

II2 Paus. II, II, 3 .

II3 Paus. VIII, 54, 5 .

II4 Sur Dionysos Mystès, voir Jost, Sanctuaires, op. cit. (note 2) 435-436.

II5 Paus. IX, 24, 5 . 
à Épidaure ${ }^{\mathrm{II} 6}$ et celui de Zeus à Olympie. ${ }^{\mathrm{II7}}$ Pausanias précise même à leur propos que l' $\not \chi \lambda \sigma o \varsigma$ est ífóv, soulignant ainsi le caractère particulièrement vénérable des lieux.

La description de ce que Pausanias appelle "l'alsos de Trophonios" montre néanmoins la difficulté d'apprécier à leur juste mesure les emplois du mot. En effet, après avoir par deux fois désigné le sanctuaire de cette manière, ${ }^{\text {II8 }}$ il précise que le

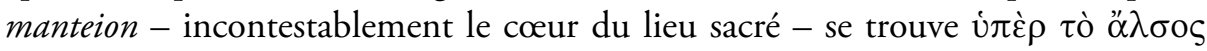

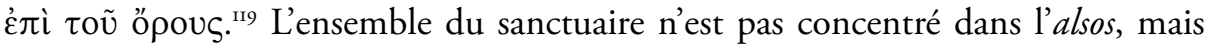
l'importance de ce dernier est telle qu'il a donné son nom au tout. De la même manière, le sanctuaire des Muses de l'Hélicon est constamment désigné par Pausanias du nom d'alsos des Muses. ${ }^{120}$ Ces sanctuaires pourraient donc tout autant s'inscrire dans la cinquième et dernière catégorie qu'il s'agit à présent d'évoquer.

(5) L'alsos peut s'élever dans une partie seulement d'un ensemble plus vaste et ne pas se confondre avec le sanctuaire. À soixante stades de Pellène en Achaïe se trouve le Mysaion, un sanctuaire de Déméter Mysia, et dans ce Mysaion s'élève un alsos aux arbres variés et à la source d'eau vive. ${ }^{121}$ De même, dans l'enceinte des

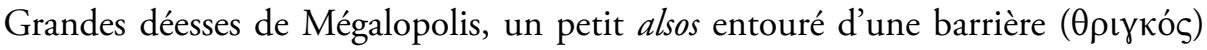
est inaccessible. Dans le sanctuaire de Despoina à Lykosoura en Arcadie, enfin, au-

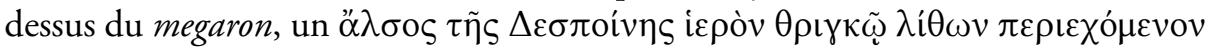
renferme une curiosité botanique : un olivier et un pin puisant naturellement leur substance à la même racine. ${ }^{122}$ Le fait que ces trois descriptions concernent l'intérieur d'un sanctuaire de Déméter ou de sa fille relève tout d'abord de l'intérêt particulier de Pausanias pour ces déesses, et donc de son souci de précision quand aucun interdit ne le contraint au silence. Ces évocations soulignent aussi les caractéristiques topographiques particulières aux sanctuaires à vocation mystérique des deux déesses. ${ }^{123}$ D'autres divinités que Déméter voient leur alsos frappé d'un interdit ou dissimuler un objet de culte invisible au profane. Ainsi, l'alsos d'Arès à Geronthrai est interdit aux femmes pendant la fête annuelle. ${ }^{124}$ Dans celui d'Artémis Sôteira

II6 Paus. II, 27, I ; 27, 5; 27, 7.

II7 Paus. V, Io, I. L'appellation conventionnelle d'Altis serait une déformation du mot $\alpha$ $\lambda \sigma o \varsigma$ :

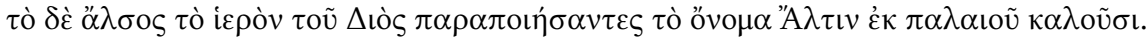
Cf. Casevitz, "Temples et sanctuaries ", loc. cit. (note 4) 93, note 78.

II8 Paus. IX, 39, $2 ; 39,4$.

II9 Paus. IX, 39, 9.

I2O Paus. IX, 29, 5 (2 occ.) ; IX, 3I, 3 (2 occ.).

I2I Paus. VII, 27,9.

I22 Paus. VIII, 37, IO.

I23 Paus. II, 22, 3 ; VIII, 3I, 5 ; VIII, 36, 6 ; IX, 25, 5. Cf. Pirenne-Delforge, Retour à la source, op. cit. (note I) chap. VI.

I24 Paus. III, 22, 6-7. 
à Pellène ne peuvent pénétrer que les prêtres ${ }^{125}$ et la statue d'Héra dans l'alsos de la déesse à Aigion n'est visible que par la prêtresse. ${ }^{\mathrm{I} 2}$

L'alsos est un lieu naturellement circonscrit dans le paysage, où la présence divine a pu être ressentie avec une intensité particulière. ${ }^{127}$ Pausanias est très sensible au charme de ces endroits ombragés et frais auxquels arbres et sources conferent une ancienneté tangible, indépendamment des constructions humaines. Hormis l'alsos d'Argos le Niobide, dont il ne parle que dans les récits sur le sacrilège de Cléomène de Sparte, ${ }^{128}$ Pausanias ne décrit d'alsos qu'en référence à des dieux ou, dans le cas de Trophonios, à un héros dont le culte est manifestement divin, ${ }^{129}$ à l'instar de celui d'Héraclès ou d'Asclépios. Jamais les groupements d'arbres autour d'un tombeau héroïque ne reçoivent le nom d'alsos sous sa plume. ${ }^{130}$

Dans le cas de l'Altis, le nom d'alsos relève autant de la convention que de la réalité du paysage. Pausanias se fait l'écho d'un usage. Établir une telle distinction pour d'autres lieux n'est guère aussi aisé. Ainsi, de vieux cyprès poussent dans l'Asclépieion de Titanè et des oliviers dans celui d'Épidaura Limera. Une frondaison touffue de cyprès entoure le sanctuaire d'Eurynomè à Phigalie et des palmiers poussent devant le sanctuaire d'Artémis à Aulis. ${ }^{33}$ Les oliviers peuvent éventuellement appartenir à des parcelles dont l'affermage permettait d'accroître les revenus du sanctuaire et l'exploitation du bois d'autres arbres pouvait également y contribuer. ${ }^{132}$ Dans ce dernier cas, l'emploi du terme d'alsos, avec sa dimension religieuse, ne devait pas correspondre, dans la perspective de Pausanias, aux réalités du terrain.

I25 Paus. VII, 27, 3 .

I26 Paus. VII, 23, 9 .

I27 P. Bonnechere fait l'hypothèse quel' alsos était " a natural and divine manifestation of a median place between two worlds": "The place of the sacred grove ", loc. cit. (note 84) 4I.

I28 Paus. II, 20, 8 ; III, 4, I. Cf. Birge, «Trees ", loc. cit. (note 85) 238.

I29 Paus. IX, 39, 2 et 4. La même explication a été avancée pour l'attribution de hiera ou de naoi à des personnages que nous qualifions d'héroïques. Cf. supra, p. I50. - Dans le cas du temenos de Bellérophon dans l'alsos Kraneion de Corinthe (II, 2, 4), il entre dans la première catégorie développée ci-dessus et ne justifie pas de qualifier l'alsos d'héroïque (ce que fait Jacob, "Paysage et bois sacré ", loc. cit. [note 84] 36).

I30 E. g. Paus. I, 42, 7 ; II, 28, 7 ; IV, I, 5-6 ; V, I3, I ; VIII, 35, 8. Cf. Birge, "Trees ", loc. cit. (note 85) 236-237. - Des textes contemporains associent pourtant le terme d'alsos à un tombeau héroïque, ce qui laisse penser que Pausanias a fait ce choix sciemment : e.g. Inscriptiones Graecae $\mathrm{II}^{2}$, 3819 (époque impériale), pour un philosophe. Je remercie Pierre Bonnechere pour cette indication.

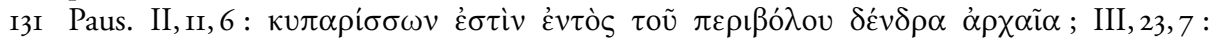

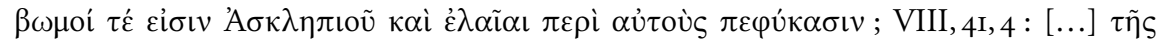

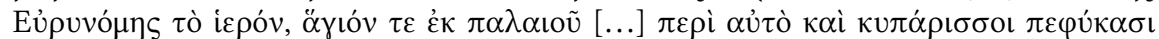

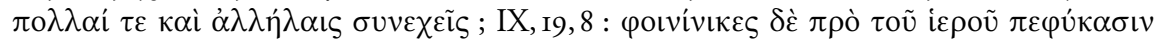
$[\ldots]$.

I32 M. P. J. Dillon, "The Ecology of the Greek Sanctuary », Zeitschrift für Papyrologie und Epigraphik II8 (1997) II3-I27, spéc. II6-II9; G. Ragone, "Dentro l'’ $\lambda \sigma o s$. Economia e tutela 
Mais une telle hypothèse ne peut s'appliquer systématiquement aux différents lieux boisés d'un sanctuaire dont le visiteur ne fait pas un alsos. ${ }^{133}$

\subsection{Té $\mu \varepsilon v o \varsigma^{134}$}

Si le terme n'eut pas, à l'origine, une signification religieuse, ${ }^{135}$ c'est pourtant une telle connotation - et rien qu'elle - que reçoit le mot dans les descriptions et les évocations de Pausanias. La notion de "découpage ", présente dans son sens étymologique, permet de comprendre bon nombre d'emplois de temenos par rapport à ceux de hieron : c'est l'espace sacré, le terrain réservé à un dieu ou à un héros qui est ainsi mis en évidence, et pas seulement la sacralité du lieu. De surcroît - contrairement aux doutes que suscite de ce point de vue l'usage de hieron -, la présence d'un naos n'est pas une hypothèse nécessaire en l'absence d'une référence explicite.

À Athènes, le grand sanctuaire de Zeus Olympien accueille bon nombre d'antiquités, un Zeus en bronze, un naos de Cronos et Rhéa, un temenos de la Terre Olympienne. ${ }^{136} \mathrm{C}$ 'est à cet endroit qu'une ouverture d'une coudée dans le sol aurait absorbé les flots du déluge. Le temenos est ici un espace non bâti, clairement délimité au sein d'un ensemble plus vaste. L'explication par le souci stylistique d'une variatio sermonis ${ }^{137}$ (bieron de Zeus, naos de Cronos et Rhéa, temenos de Gè) n'est peut-être pas absent du choix de désigner ainsi le sanctuaire de Gè, mais l'espace non construit qui accueille une faille ancestrale reçoit de la sorte une dimension davantage explicite de domaine réservé à la puissance primordiale de la Terre au sein même du sanctuaire de Zeus.

del bosco sacro nell'antichità classica ", in : C. A. Livadie - F. Ortolani, Il sistema uomoambiente tra passato e presente (1998) $\mathrm{II}-25$.

I33 Pour une tentative de classer les caractéristiques de l'alsos chez Pausanias, voir Birge, "Trees", loc. cit. (note 85) 240-245.

I34 Sur 58 occurrences, 44 emplois s'inscrivent dans une description et concernent 34 endroits différents, Io fois le mot est employé hors de toute description pour évoquer io lieux différents, et il apparaît également dans 3 citations.

I35 Casevitz, "Temples et sanctuaires », loc. cit. (note 4) 85-87: "On explique par les exemples anciens comment un temenos a pu se spécialiser au sens d'enceinte consacrée : c'est à l'origine une terre prélevée pour honorer et assurer les revenus d'un homme puis d'un dieu " (p. 87). Cf. W. Donlan, "Homeric temenos and the Land Economy of the Dark Age", Museum Helveticum 46 (1989) I29-I46.

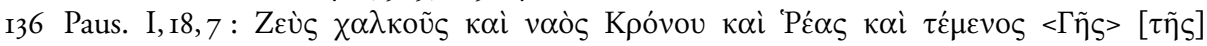

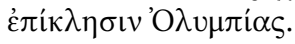

I37 Une occurrence de temenos semble relever d'un tel souci : dans le récit de la pétrification de la prêtresse d'Athéna Itonia, Pausanias utilise temenos juste après avoir désigné le sanctuaire à l'aide des termes hieron et naos (IX, 34, 2). Cf. aussi IX, IO, 5 . 
La topographie à Olympie est pareillement complexe. Dans l'Altis, les cultes sont nombreux. Un héros s'y distingue pourtant par l'importance de son culte : Pélops. ${ }^{138}$ Le Pélopion, comme l'identifie Pausanias, est un espace réservé dans le

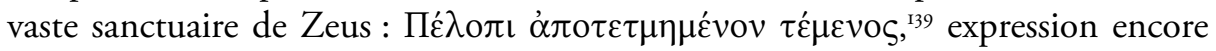
renforcée par la description du mur de pierres qui ceint le temenos. ${ }^{140}$ Inversement, dans deux cas, Hippolyte à Trézène ${ }^{\mathrm{I41}}$ et Apollon à Delphes, ${ }^{\mathrm{I} 42}$ temenos désigne le sanctuaire dans toute la complexité d'une implantation "polythéiste ". C'est par

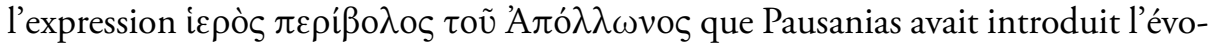
cation du sanctuaire delphique dont il loue tant la grandeur que la localisation particulière. ${ }^{143}$ Quant à la description proprement dite, elle commence dès l'entrée dans le temenos, dans le domaine réservé au dieu pourvoyeur d'oracles. ${ }^{144}$

Le sanctuaire de Zeus Lykaios sur le mont du même nom est représentatif de ces lieux de la chôra qui inscrivent dans le paysage un espace consacré à un dieu. Parmi les merveilles du mont Lycée, Pausanias place en tête le temenos inaccessible de Zeus. Or, à quatre reprises dans le même paragraphe, il l'évoque sous ce nom, ${ }^{145}$ ce qui donne à penser qu'il n'a pas sacrifié au style la technicité de son vocabulaire. ${ }^{\mathrm{I} 46}$ Les fouilles ont révélé une structure assez vaste $(55 \times$ I20 $\mathrm{m})$ qui ne semble pas avoir accueilli de temple. ${ }^{147}$ En revanche, dans ce cas précis, le terme de temenos ne désigne pas l'ensemble du sanctuaire : le tertre de terre monumental qui fait office d'autel se situe au sommet de la montagne, à l'extérieur du temenos.

Dans d'autres régions visitées par Pausanias, semblables temenè, hors-les-murs des cités, dépourvus de temple et vraisemblablement délimités par une enceinte, ont retenu l'attention du visiteur : dans la plaine sur la route du Taygète en Laconie, le temenos de Zeus Messapien, ${ }^{148}$ celui de Kranios Stemmatios sur la route de

I38 Paus. V, I3, I.

I39 Ibid.

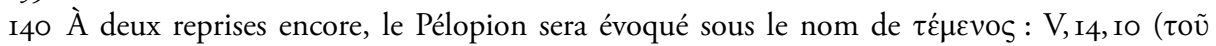

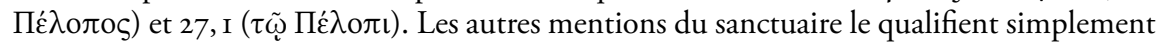
de Pélopion $(\mathrm{V}, \mathrm{I} 3, \mathrm{I} ; \mathrm{I} 3,8 ; 24,5)$.

I4I Paus. II, 32, I.

I42 Paus. X, 9, 3 .

I43 Paus. X, 9, I.

I44 En X, 37, 6, Pausanias se fait l'écho d'un oracle qui désigne le sanctuaire delphique d'Apollon par le terme de temenos, que le Périégète paraphrase en utilisant le même mot. De même, l'épigramme d'un ex-voto de l'Altis désigne le sanctuaire de Zeus à Olympie comme temenos (VI, 3, I4).

I45 Paus. VIII, 38,6 .

I46 Même en dehors d'une description, le sanctuaire arcadien est temenos : IV, 22, 7.

I47 Jost, Sanctuaires, op. cit. (note 2) I8O-I8I.

I48 Paus. III, 20, 3. 
Sparte vers l'Arcadie, ${ }^{\mathrm{I} 49}$ le temenos des Néréides au bord de la côte laconienne, ${ }^{\mathrm{I} 0}$ celui de Télèphe sur le mont Parthénion en Arcadie, ${ }^{15 \mathrm{IS}}$ le temenos de Zeus Laphystios entre Koronée et le mont Laphystion en Béotie. ${ }^{152}$

L'utilisation du terme n'est cependant pas exclusive des enceintes extraurbaines. Pausanias énumère des temenè dans les cités ou à leur périphérie immédiate. Ainsi, à Myonia en Locride, un temenos appelé Poseidonion et situé útè $\rho$

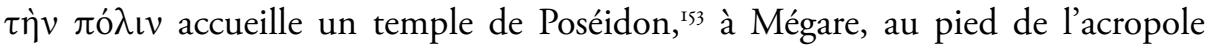
Karia, l'Olympieion, un temenos de Zeus, abrite également un temple digne d'attention. ${ }^{154}$ En face de l'agora de Patras, la structure est plus complexe : le temenos d'Artémis accueille un naos de la Limnatis, mais d'autres hiera s'y trouvent, associés à une structure de portiques. ${ }^{155}$

Hormis ces sanctuaires, au sein desquels Pausanias signale la présence d'un temple, les temenè urbains ne semblent pas plus que les grandes enceintes de la chôra avoir accueilli de naos. Le sanctuaire d'Athéna Sôteira et Zeus Sôter au Pirée était le plus important du port athénien. ${ }^{156}$ Pausanias y décrit la statue de chacune des deux divinités du lieu et évoque la présence de peintures. ${ }^{157}$ Au centre d'Athènes,

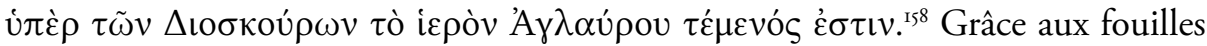
de G. Dontas, le sanctuaire d'Aglauros est aujourd'hui localisé avec certitude près du péripatos de l'Acropole en son flanc ouest. ${ }^{599}$ Le temenos en question était donc

I49 Paus. III, 20, 9. Ce Kranios semble être une version locale d'Apollon Karneios : D. Musti - M. Torelli, Pausania. Guida della Grecia. Libro III : La Laconia (I99I, I9922) 259.

I50 Paus. III, 26, 7. En II, I, 8, Pausanias décrit le socle d'une des statues du sanctuaire de Poséidon sur l'Isthme où apparaissent les Néréides. Saisissant l'occasion de cette mention, il précise que ces déesses reçoivent des honneurs en maintes places et que, notamment, on leur dédie des temenè près des ports. - À Patras, près du port, un temenos d'Aphrodite accueillait une statue acrolithe de la déesse (VII, 2I, IO).

I5I Paus. VIII, 54, 6.

I52 Paus. IX, 34, 5. - Le sanctuaire de Zeus sur le mont Ithomè en Messénie ne fait pas l'objet d'une description directe, mais se voit évoqué à maintes reprises au fil des récits sur les guerres avec Sparte qui occupent la majeure partie du livre IV. Pausanias use une fois du mot temenos (IV, 3, 9) et deux fois de hieron (IV, I2, $8 ; 33, \mathrm{I})$ pour le désigner.

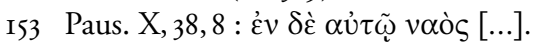

I54 Paus. I, 40,4 et 6.

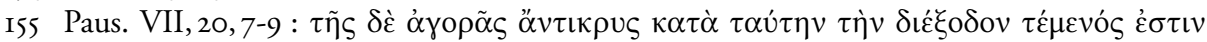

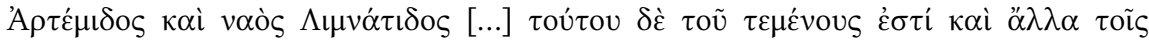

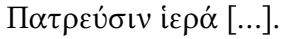

I56 I, I,3. Cf. D. Musti - L. Beschi, Pausania. Guida della Grecia. Libro I : L'Attica (1982, I995 $5^{4}$ 254. - Un temenos était consacré au même Zeus Sôter, avec des agalmata, à Aigion (VII, 23, 9).

I57 Strabon (IX, I5 [C396]) évoque pareillement le hieron accueillant de petits portiques avec des peintures et des statues à l'air libre.

I58 Paus. I, I8, 2.

I59 G. Dontas, "The True Aglaurion », Hesperia 52 (1983) 48-63. 
un espace réduit, ménagé pour le culte en fonction du relief rocailleux du flanc de la citadelle. C'est bien la notion d'espace consacré que souligne l'emploi de temenos, sans qu'un temple soit seulement envisageable dans ce cas. Dans la montée de l'Acrocorinthe, Pausanias mentionnera aussi des temenè, deux pour Isis et deux pour Sarapis, sans doute des espaces du même type que celui d'Aglauros à Athènes, réservés au culte des divinités égyptiennes, clairement délimités mais sans structure bâtie d'envergure. ${ }^{160}$

À Sparte, sur l'Aphétaïs, une des grandes artères de la cité, se trouvait un temenos de Poséidon du Ténare, rappel citadin du célèbre sanctuaire du cap ainsi dénommé. ${ }^{61} C^{\prime}$ 'est de cette manière aussi qu'était désigné le sanctuaire urbain de Zeus Lykaios à Mégalopolis. ${ }^{162}$ Néanmoins, dans le cas de Poséidon Tainarios, le sanctuaire de la chôra n'est pas appelé temenos: il s'agit d'un naos en forme de grotte. ${ }^{163}$ L'enceinte de l'Aphétaïs accueillait Poséidon dans la cité sans proposer l'image fidèle de son sanctuaire du bord de mer. À la sortie de Mégalopolis, les habitants sacrifiaient chaque année à Borée dans son temenos pour lui rendre grâce du salut accordé lors d'un conflit avec les Spartiates. ${ }^{164}$

Les autres temenè évoqués par Pausanias dans les cités et à leurs abords immédiats sont majoritairement dédiés à des héros et à Asclépios. Ce dernier se voit attribuer un temenos à Argos, ${ }^{165}$ dans la cité d'Épidaure, ${ }^{166}$ à Aigion, ${ }^{167}$ à Mégalopolis. ${ }^{168}$ Quant aux temenè de héros, Lakios en possède un sur la voie sacrée athénienne, ${ }^{169}$ Bellérophon aux abords de Corinthe, ${ }^{170}$ un héros anonyme proche de Dionysos à Sparte, ${ }^{171}$ une femme indigène également proche de Dionysos à Patras, ${ }^{172}$ Phylakos à Delphes. ${ }^{173}$ Est encore évoqué, lorsque Pausanias mentionne le hèrôon de Persée

I60 Paus. II, 4, $6:$ un temenos d'Isis Pelagia, l'autre d'Isis Égyptienne, un temenos de Sarapis év

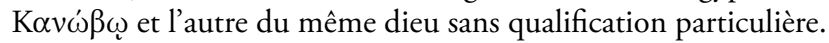

I6I Paus. III, I2, 5.

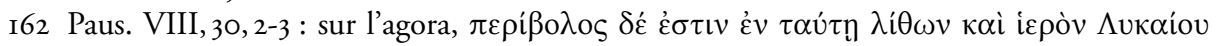

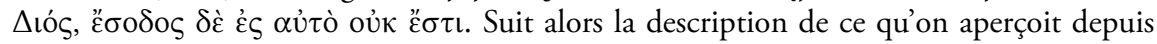

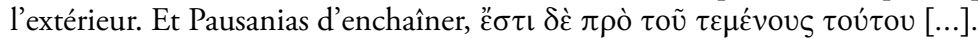

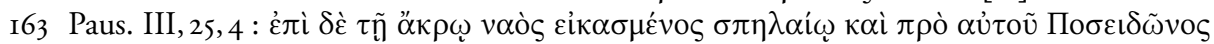
$\not ̈ \gamma \alpha \lambda \mu \alpha$.

I64 Paus. VIII, 36, 6. Pour le récit de cette victoire, cf. VIII, 27, I4.

I65 Paus. II, 23, 2.

I66 Paus. II, 29, I.

I67 Paus. VII, 23, 7.

I68 Paus. VIII, 32, 4.

I69 Paus. I, 37, 2.

I70 Paus. II, $2,4$.

I7I Paus. III, I3, 7 .

I72 Paus. VII, 2I, 6 .

173 Paus. $\mathrm{X}, 8,7$. 
sur la route de Mycènes, le temenos du héros à Athènes. ${ }^{174}$ Rappelons également le Pélopion d'Olympie mentionné plus haut. Des enceintes en l'honneur de héros aux statuts divers recevaient donc volontiers le nom de temenos quand elles étaient dépourvues de temples - et sans doute aussi de tombeau, du moins dans l'appréciation que Pausanias fait des lieux.

Quelques derniers exemples vont nous aider à mieux cerner encore les différentes applications de la notion chez Pausanias.

À Athènes, la maison qui avait abrité le scandale de la parodie des mystères d'Éleusis à la fin du v viècle est du temps de Pausanias consacrée à Dionysos. Et Pausanias d'enchaîner, "après le temenos de Dionysos [...] ».775 De la même manière, l'oikia du tyran Cléon à Sicyone est devenue un temenos réservé aux empereurs romains. ${ }^{176}$ Ces deux maisons "profanes" passées intégralement au domaine "sacré » reçoivent dans leur nouveau statut l'appellation de temenos : une fois encore la " coupure » dans l'espace est mise en évidence. ${ }^{177}$ Un tel souci apparaissait dans la description du Pélopion à Olympie ; on le retrouve dans le récit des honneurs rendus par les Argiens à Dionysos après sa guerre contre Persée. En effet, si c'est bien un naos que Pausanias voit dans la cité, dans le récit, c'est un $\tau \dot{\varepsilon} \mu \varepsilon v o \varsigma$

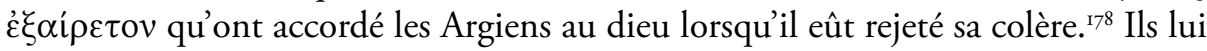
ont donné, entre autres honneurs d'envergure, une parcelle du territoire qui lui appartient désormais en propre. Cet exemple évoque la signification ancienne du temenos comme "domaine prélevé, réservé hors de l'ensemble des terres ». ${ }^{179}$

Pour terminer, venons-en à la visite de Pausanias sur l'agora d'Élis. ${ }^{180}$

I74 Paus. II, I8, I. - Dans le récit des aventures d'Euthymos de Locres à Témessa, Pausanias rapporte un oracle de la Pythie qui ordonna aux gens du lieu de se rendre le daimôn Hérô

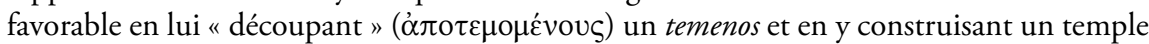
(VI, 6, 8).

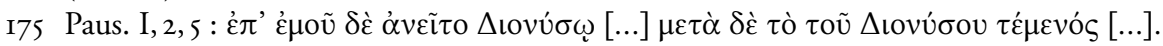

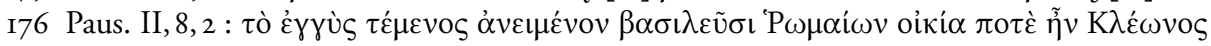

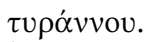

I77 À Thèbes, la maison de Kadmos et de ses descendants serait devenue le hieron de Déméter Thesmophoros (IX, I6, 5).

I78 Paus. II, 23, 7.

I79 Casevitz, "Temples et sanctuaires ", loc. cit. (note 4) 86 ; Donlan, "Homeric temenos ", loc. cit. (note 135$)$ I 45 .

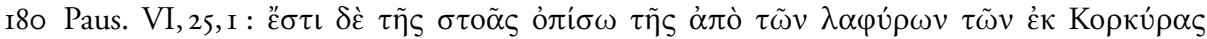

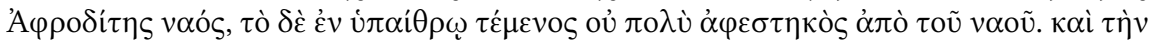

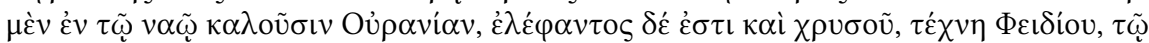

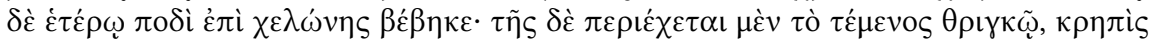

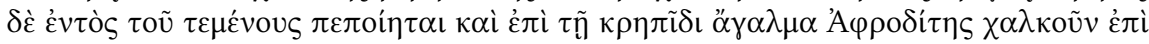

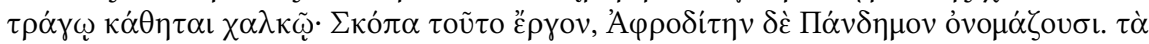

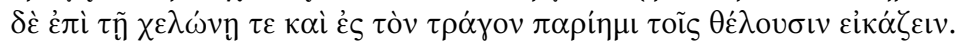


Derrière le portique construit avec le butin pris à Corcyre, il y a un temple d'Aphrodite et, se trouvant non loin du temple, le temenos à l'air libre. Celle qui se trouve dans le temple, ils l'appellent Ourania, elle est en ivoire et en or, et due à l'art de Phidias; elle pose l'un des deux pieds sur une tortue. Le temenos de l'autre est entouré d'un mur. À l'intérieur du temenos se trouve une krepis et, sur la krepis, une statue d'Aphrodite en bronze est assise sur un bouc en bronze. L'œuvre est de Scopas et ils nomment Aphrodite Pandèmos. Quant à la signification de la tortue et du bouc, je laisse à ceux qui le désirent le soin de la conjecturer.

Il s'agit donc manifestement d'un sanctuaire d'Aphrodite accueillant deux statues de la déesse, diversement qualifiées : l'Ourania de Phidias s'élève dans le temple, la Pandèmos de Scopas se situe dans le temenos, qui sert en quelque sorte d'écrin à cette seule statue et non d'enceinte englobant la totalité du sanctuaire. ${ }^{18 \mathrm{r}}$ De la même manière, le temenos extra-urbain de Zeus Lykaios était indépendant de l'autel.

L'utilisation du mot temenos dans la Périégèse ne paraît donc pas, au terme de ce parcours, relever d'un choix arbitraire de l'auteur ou du seul souci de travailler son style en variant son vocabulaire. Temenos désigne un espace consacré dont la délimitation est manifeste, et qui, sans autre précision dans la description, ne semble pas avoir accueilli de temple. Il sert aussi à désigner un bâtiment qui est passé d'un usage profane à un usage sacré, comme dans le cas de la maison où les mystères d'Éleusis avaient été parodiés à Athènes et dans celui de la maison du tyran Kléon à Sicyone. Dans un tiers des occurrences, il est utilisé pour désigner le lieu de culte d'un héros. Là encore, la notion de " domaine réservé " pourrait être entrée en résonance avec les emplois anciens du terme de temenos qui concernaient les figures royales de l'épopée. C'est peut-être la raison pour laquelle Pausanias a ainsi qualifié le sanctuaire athénien d'Aglauros : ni hieron (qui est avant tout divin, nous a-t-il semblé), ni hèrôon (puisqu'il n'enferme aucune tombe), le lieu de culte de la princesse athénienne était tout simplement l'espace que la cité lui réservait pour l'honorer : un temenos, un domaine circonscrit au flanc de la prestigieuse citadelle. Quant aux temenè divins, qu'ils fussent ou non construits, c'est sans doute davantage leur apparence qui devait faire surgir l'appellation, celle d'un espace bien délimité, ainsi que va davantage encore l'évoquer l'utilisation du mot $\pi \varepsilon \rho i ́ \beta o \lambda \circ \varsigma$ en alternance avec temenos.

I8I Sur ces deux statues, voir V. Pirenne-Delforge, "Des épithètes exclusives dans la Grèce polythéiste ? L'exemple d'Ourania ", in : N. Belayche - P. Brulé - G. Freyburger - Y. Lehmann - L. Pernot - F. Prost (éds.), Nommer les dieux. Théonymes, épithètes, épiclèses dans l'Antiquité (2005) 27I-290, spéc. 283-286. 


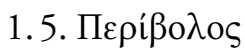

Le mot, qui désigne une enceinte, n'appartient pas à proprement parler au vocabulaire religieux. Ainsi, sur 70 occurrences, I4 désignent des remparts, ${ }^{182}$ quatre des enclos où s'exercent des athlètes ${ }^{1{ }^{183}}$ deux des enceintes d'agora. ${ }^{184}$ Toutefois, la prise en considération du mot dans le contexte qui nous occupe n'est pas négligeable puisque 50 emplois entrent en ligne de compte pour la description de sanctuaires. ${ }^{\mathrm{I} 85}$

Le peribolos désigne aussi bien la vaste enceinte d'un sanctuaire important ${ }^{186}$ que les quelques pierres qui enserrent une béance réputée pour ses liens avec l'au-delà. ${ }^{87}$ Le choix du terme relève d'un souci d'exactitude dans la description puisqu'il fait référence à une réalité topographique tangible ; il articule également la présentation des diverses composantes d'un sanctuaire. Ainsi, près du théâtre d'Athènes s'élève, au dire de Pausanias, le plus ancien hieron de Dionysos ${ }^{188}$ et à l'intérieur de son peribolos se trouvent deux temples, l'un pour le Dionysos d'Éleuthères, l'autre abritant une réalisation d'Alkamène. Le propos passe donc graduellement du générique d'un lieu sacré au particulier des statues de culte. La référence au peribolos permet de situer les temples jumeaux dans une même structure sans recourir à nouveau à l'emploi de hieron.

I82 Paus. I, 25, 8 ; 4I, 6 ; II, 3, 3 ; I6, 5; IV, 9, 2 ; 27, 7 ; VI, I9, II ; VII, I8, 5 (2 occ.) ; VIII, I3, 2 ; $38, \mathrm{I} ; \mathrm{IX}, 7,4 ; 8,4 ; \mathrm{X}, 4,2$.

I83 Paus. VI, 2I, $2 ; 23$, I et $4-5$.

I84 Paus. VII, 22, 2 ; X, 32, IO.

I85 46 emplois s'inscrivent dans une description et concernent 3I endroits différents, 4 fois le mot est employé en dehors d'une description. Pour les descriptions, il faut préciser que près de la moitié des periboloi apparaissent au livre II (2I occurrences pour I4 lieux).

I86 Paus. I, I8, 6 et 7 : Zeus Olympien à Athènes; II, 2, I : Poséidon sur l'Isthme; II, 27, I et 3 : l'alsos sacré d'Asclépios à Épidaure; II, 33,3: Poséidon à Kalaurie; V, 4, 8 ; I5, 2 ; 27, II : Zeus à Olympie; VIII, 3I, I et 4 et 5 et 7 : les Grandes déesses à Mégalopolis; VIII, 37, I et 3 : Despoina à Lykosoura; X, 9, I ; 32, I : Apollon à Delphes ; X, 32, I2 : Asclépios à Tithoréa ; X, 32, I3 : Isis à Tithoréa. - Pour désigner l'intérieur du sanctuaire de Déméter à Éleu-

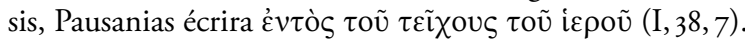

I87 Paus. II, I5, 3 : dans le district de Némée, le tombeau d'Opheltès est une béance entourée d'un mur de pierres intégrant des autels; Paus. II, 36,7 : aux environs de Lerne, un peribolos de pierres désigne le lieu où Plouton a enlevé Korè ; IX, 8,3: sur la route de Potniai à Thèbes. À ces diverses enceintes, on peut ajouter le peribolos du manteion de Trophonios

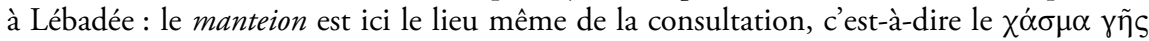
artificiel par lequel descendent les consultants et autour duquel devait s'élever une barrière (IX,39, 9), et peut-être même des periboloi où s'accomplissent de mystérieux rituels en l'honneur de Déméter à Hermione (II, 34, IO). Soulignons enfin, pour clore cette énumération, qu'à Élis, Hadès a un hieros peribolos et un naos, affirmation que Pausanias répète afin d'en souligner le caractère exceptionnel (VI, 25, 2).

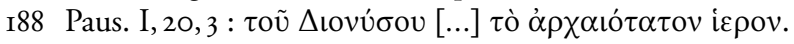


À l'instar de ses temenè, les periboloi d'Asclépios sont plus nombreux que pour n'importe quelle autre divinité : à Sicyone, ${ }^{189}$ à Titanè ${ }^{190}$ à Épidaure, ${ }^{191}$ à Tithoréa, ${ }^{192}$ ce qui tend à confirmer la similitude de caractère des lieux sacrés que Pausanias désigne du nom de temenos et de hieron enclos, à l'exception de la présence d'un temple qui est assurée dans les quatre periboloi. Cette similitude est encore attestée par la description du sanctuaire urbain de Zeus Lykaios, sur l'agora de Mégalopolis. ${ }^{193}$ Pausanias y a vu un peribolos de pierres et un hieron du dieu dont l'entrée est interdite. Il décrit néanmoins ce qu'il y voit de l'extérieur, ce qui laisse clairement entendre que le hieron n'est pas, dans ce cas, un naos. Il clôt sa description par la mention du temenos de Zeus, appellation générique du hieron enclos d'un peribolos qu'il attribue explicitement au dieu six paragraphes plus loin. ${ }^{194}$ Cet exemple vient confirmer l'interprétation du hieron d'Héraclès donnée plus haut : ${ }^{195}$ le mot hieron désigne tout d'abord l'ensemble du sanctuaire, que Pausanias détaille par l'énumération du peribolos Paidizè, dans lequel se trouve le hieron qui, cette fois, est le temple dans lequel s'élève le xoanon. ${ }^{196}$ C'est donc bien tout le peribolos qui est le hieron d'Héraclès, comme le peribolos de l'agora mégalopolitaine est consacré à Zeus.

\section{Les sanctuaires particuliers ou spécialisés}

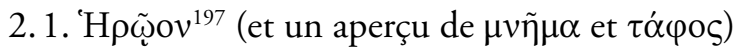

La signification du terme hèrôon est donnée par sa formation même. Il s'agit du lieu où l'on honore un être humain auquel sa mort a conféré un statut héroïque, qu'il s'agisse d'un personnage épique ou d'un individu dont l'historicité n'est pas contestable. ${ }^{198}$

I89 Paus. II, IO, 2 et 4 .

I9O Paus. II, I, 6 et 8.

I9I Paus. II, 27, I et 3.

192 Paus. X, 32, 12.

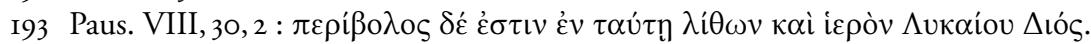

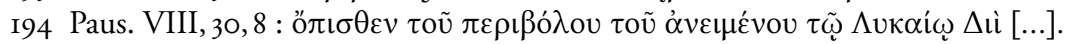

I95 Cf. supra, note 4.

I96 Paus. II, IO, I.

197 Sur 42 occurrences, 39 emplois s'inscrivent dans une description (7 occurrences apparaissent au pluriel) et concernent 36 endroits différents, 3 fois le mot est employé en dehors d'une description.

198 Une telle dichotomie n'est évidemment pas le fait de Pausanias pour qui l'historicité d'Agamemnon ou de Persée n'est pas douteuse. 
Mais l'utilisation d'un vocabulaire diversifié pour désigner le sanctuaire d'un héros (bieron, naos, temenos) laisse penser que le hèrôon est une structure dont la spécificité ne tient pas seulement à la qualité héroïque de celui qui s'y voit honoré. Pausanias n'explicite guère ce qu'il entend exactement par une telle évocation et se limite le plus souvent, pour qualifier le lieu du culte héroïque, à déterminer le mot hèrôon du nom de son propriétaire. Il précisera parfois, çà et là, que le hèrôon d'Ino à Mégare est entouré d'un mur de pierres et que des oliviers y poussent aussi, ${ }^{199}$ que celui d'Hyrnetho dans la région d'Épidaure comprend des oliviers dont il est interdit d'emmener les branchages, même tombés à terre, ${ }^{200}$ ou bien que les hèrôa des fils de Psophis, l'éponyme de la ville arcadienne, n'étaient plus fameux de son temps. ${ }^{20 I}$ Une lecture attentive permet néanmoins de confirmer ce que le statut héroïque laissait déjà entendre : la mort étant à l'origine des honneurs rendus, c'est une tombe, réelle ou supposée, qui détermine l'établissement d'un hèrôon. Plusieurs exemples l'attestent. Ino et Iphigénie possèdent chacune un hèrôon à Mégare car, nous dit Pausanias, elles auraient été inhumées en ce lieu ; ${ }^{202}$ Aigialeus, fils d'Adraste, est enterré à Pagai et son hèrôon s'appelle encore Aigialeion ; ${ }^{203}$ de la même manière, Aratos a été enterré à Sicyone et son hèrôon porte le nom d'Arateion ;204 les frères d'Hyrnetho ont emmené le cadavre de leur sœur à l'endroit désormais appelé Hyrnethion et ont établi un hèrôon. ${ }^{205}$ Une confirmation a contrario est apportée par la mention du hèrôon d'Iolaos à Thèbes: Pausanias l'assortit d'une réserve puisque les Thébains eux-mêmes admettent qu'il est mort en Sardaigne, ${ }^{206}$ preuve que le hèrôon implique la mort, ou du moins l'ensevelissement, sur place. L'exemple du héros archégète de Tronis en Daulide est le plus explicite. L'identité de l'archégète en question est controversée, mais le rituel est décrit : quotidiennement les Phocidiens apportent des victimes sacrificielles dont le sang s'écoule dans une ouverture pratiquée dans la tombe. ${ }^{207}$ C'est donc le taphos qui est au centre du hèrôon.

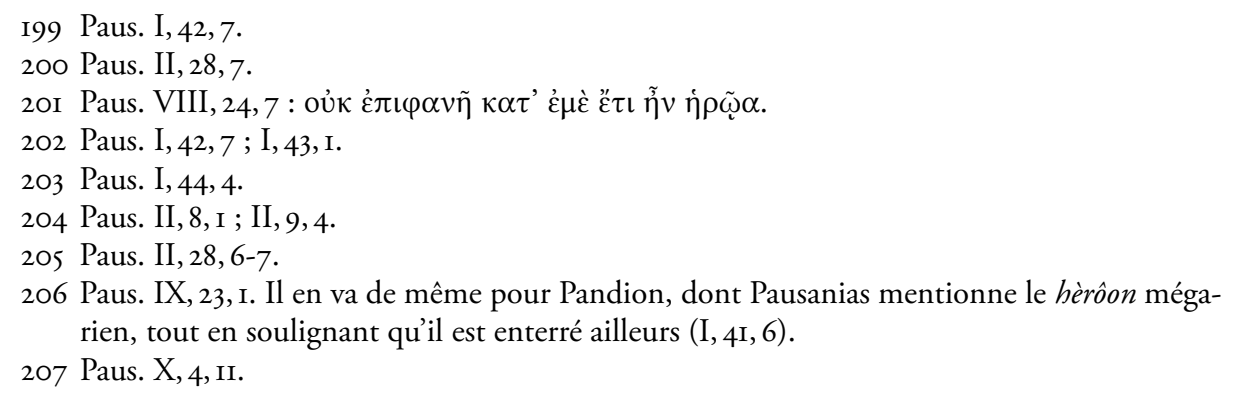


Les nombreuses tombes de héros relevées au passage par Pausanias, ${ }^{208}$ sans qu'il soit fait mention d'un hèrôon, font parfois l'objet d'un rituel particulier, ${ }^{209}$ quand ce ne sont pas leurs caractéristiques formelle ${ }^{210}$ qui reçoivent une attention spéciale dans la description. Sans prétendre qu'aucune de ces tombes ne puisse être qualifiée de hèrôon en l'absence d'une telle notation, et donc que Pausanias ait fait preuve de la rigueur la plus grande dans l'évocation de ces réalités héroïques, on est tenté de concevoir, là où il parle d'un hèrôon, l'existence d'une structure bâtie autour ou à l'entour du tombeau. Car, à une exception près, quand il parle d'un mnèma ou d'un taphos isolé, jamais une telle structure n'apparait. L'exception concerne Castor à Sparte, qui possède un $\mu v \tilde{\eta} \mu \alpha$ sur lequel un hieron a été établi. ${ }^{2 I I}$ Lexplication d'une telle structuration du lieu sacré réside dans le processus de divinisation qui a frappé les Tyndarides. ${ }^{212}$ Par analogie, on peut supposer que le hèrôon est une structure bâtie sur - ou à côté de - certaines tombes de héros pour accroître le prestige de leur statut. Et le fait que le hèrôon d'Alkathoos soit devenu un dépôt d'archives lorsque Pausanias visite Mégare viendrait confirmer cette hypothèse. ${ }^{213}$

$2082 \mathrm{I} 8$ occurrences pour $\mu \nu \tilde{\eta} \mu \alpha$ (dont 3 citations et une restitution), I63 pour $\tau \dot{\alpha} \alpha$ os, 6 pour $\sigma \tilde{\eta} \mu \alpha$ (dont 2 citations). L'utilisation respective de $\mu \nu \tilde{\eta} \mu \alpha$ et de $\tau \dot{\alpha} \varphi \circ \varsigma_{\text {ne semble pas tra- }}$ duire une différence formelle (les équivalents français "tombeau " et " tombe " seraient pareillement interchangeables) car, à de nombreuses reprises, les deux termes interviennent dans la description d'un même monument et répondent donc à des impératifs plus stylistiques que sémantiques (e.g. II, II, I ; 22, I ; V, 6, 6; VI, 2I, 3 ; VII, I7, 8 ; 25, I3 ; VIII, II, 8 ; $\mathrm{I} 3,5 ; 26,4$; IX, I7, $6 ; 22,6 ; \mathrm{X}, 24,6)$. S’il faut tout de même refléter dans la traduction la différence de terme, on peut traduire taphos par « tombe / tombeau » et mnèma par « monument funéraire ». Cf. M. Jost, in : M. Casevitz - M. Jost, Pausanias. Description de la Grèce, tome VIII. Livre VIII : L'Arcadie (1998) XLII : tombe et monument.

209 E. g. Paus. I, 4I, 9 (le tombeau de Térée à Mégare); II, 20,3 (Phoroneus à Argos); IV, 32, 3 (Aristomène à Messène); VII, I7, 8 (Sostratos peu avant Dymaion); VII, 20, 9 (Preugénès à Patras); IX, I8, 3 (les enfants d'Cdipe sur la route de Thèbes à Chalcis).

210 E. g. Paus. I, 43, 8 (le tombeau de Koroibos sur l'agora de Mégare) ; I, 44, 6 (Kar sur la route de Mégare à Corinthe) ; II, 2, 4 (Laïs dans le Kraneion aux abords de Corinthe) ; II, I5, 3 (Opheltès dans le hieron de Zeus à Némée) ; II, 29, 9 (Phokos à Égine); VIII, I6, 5 (une Hélène indigène de Jérusalem).

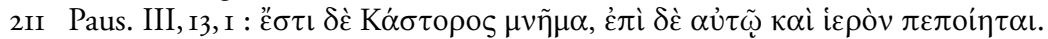

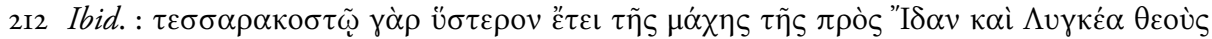

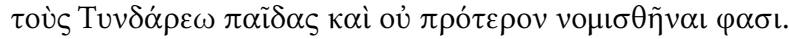

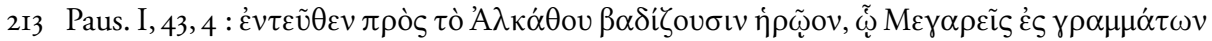

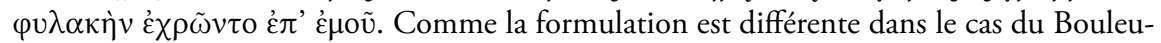
tèrion de Mégare, qui est venu surplomber des tombeaux hérö̈ques, on peut supposer que

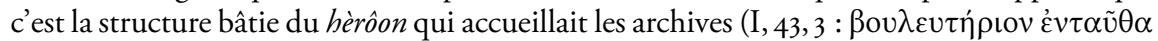

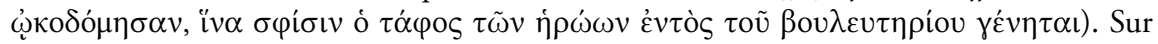
les formes diverses des hèroa, voir Hellmann, L'architecture grecque, op. cit. (note 58) 28I287. 


\section{2. 'A $\delta v \tau o v^{214}$}

À la manière de hieron, adyton désigne, par la forme neutre de l'adjectif, la qualité prédominante d'un lieu. Hieron en souligne le caractère sacré, adyton en marque l'inaccessibilité, fût-elle conditionnelle. En outre, la formation de l'adjectif luimême laisse entendre que l'endroit réservé d'accès implique d'y descendre. Et c'est bien le cas dans la Périégèse. L'adyton y apparaît comme un terme technique essentiellement appliqué à une catégorie de sanctuaires bien déterminée et peu représentée : les espaces souterrains qui dissimulent aux regards des pratiques tombant sous le coup d'un secret, d'un interdit ou procurant une révélation. C'est clair dans le cas du culte de Palaimon sur l'Isthme qui, outre un naos, compte "ce que l'on appelle un adyton " ${ }^{215}$ dont l'entrée passe sous la terre et où, "dit-on ", Palaimon a été caché. De même, à Lébadée, l'adyton sacré de Trophonios est un antre souterrain. ${ }^{216}$ À Pellène aussi, l'adyton d'Athéna s'enfonce dans la terre sous la statue de culte chryséléphantine, lui fournissant l'humidité nécessaire à sa bonne conservation $;^{217}$ quant à savoir si ce dernier adyton avait une fonction cultuelle particulière, seul le nom permettrait de le penser.

La nature de l'adyton d'Isis à Tithoréa dont Pausanias évoque longuement le culte n'est pas précisée, mais on peut en déduire le caractère souterrain par la comparaison qu'opère Pausanias avec le culte de la déesse à Koptos en Égypte : il faut " descendre " pour atteindre l'adyton d'Isis. ${ }^{218}$ Quant aux adyta des dieux katachthonioi des cités sur le Méandre en Asie Mineure, le nom de leurs propriétaires laisse entendre qu'ils se situaient également sous terre. ${ }^{219}$

Le célèbre adyton d'Apollon à Delphes surgit une seule fois dans la Périégèse et, singulièrement, il apparait hors description. En effet, c'est à la faveur de l'évocation

21423 occurrences pour 8 lieux différents. - "A $\beta \alpha \tau$ ov n'apparaît que trois fois et une seule occurrence concerne un sanctuaire : Pausanias l'utilise pour qualifier le thalamos de Sémélè à Thèbes (IX, I2,3). Cf. M. B. Hollinshead, " ‘Adyton», (Opisthodomos», and the Inner Room of the Greek Temple », Hesperia 68 (1999) I89-218.

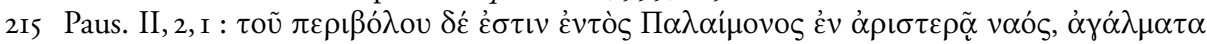

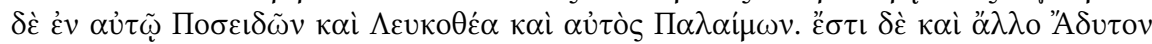

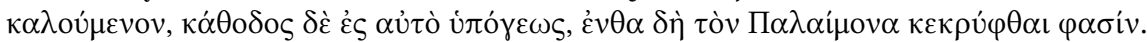

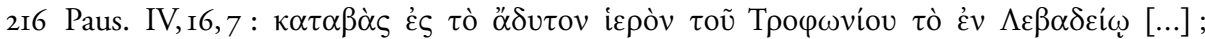

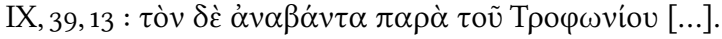

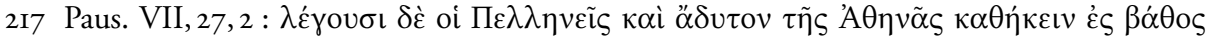

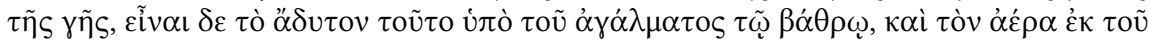

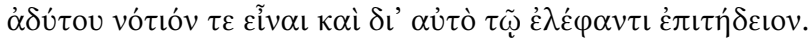

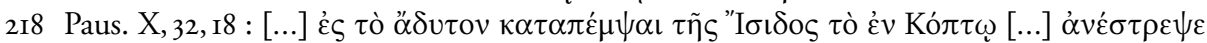
$\dot{\varepsilon} \kappa \tau \mathrm{oú} \alpha \dot{\delta} \dot{\tau} \tau \mathrm{Ou}$.

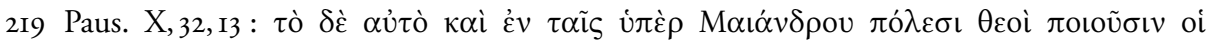

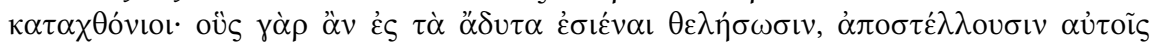

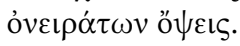


de la fontaine Kassotis que Pausanias associe adyton et inspiration divine : l'eau en question rentre sous terre et c'est elle qui « fait des femmes des prophétesses dans l'adyton du dieu ».220 En revanche, deux paragraphes plus haut, décrivant le temple lui-même, Pausanias évoque simplement «la partie la plus reculée du temple, où n'ont accès qu'un petit nombre de gens " et où se trouve une statue d'Apollon en or. ${ }^{221}$ Cette partie du temple était sans doute l'adyton, mais l'autopsie des lieux, forcément limitée par les restrictions d'accès, n'a pas fait surgir la notion dans la description. En revanche, le cours souterrain des eaux de Kassotis appelait, lui, ce nom, certes traditionnel, mais aussi techniquement adéquat pour désigner un lieu où la tradition antérieure à Pausanias précise qu’il fallait "descendre " pour consulter le dieu. ${ }^{222}$

Au vu de tous ces exemples, l'adyton de Dionysos à Amphikleia, ${ }^{223}$ pour lequel Pausanias ne donne aucun indice topographique, impliquait manifestement de "descendre " pour l'atteindre. Et il n'est pas impossible d'y voir un de ces antres dionysiaques - éventuellement souterrains - dont il convient de parler brièvement.

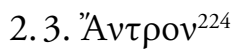

L'antre est une grotte vouée au culte d'une ou de plusieurs divinité(s), ${ }^{225}$ Dionysos s'y voit représenté sur le coffre de Kypsélos ${ }^{226}$ et, en Laconie, les gens de Brasiai ont montré au visiteur l'antre où Ino éleva le dieu ${ }^{227}$ La grotte de Déméter Melaina à Phigalie est ainsi désignée dans un oracle de Delphes ${ }^{228}$ et Pausanias use de la même appellation. ${ }^{229}$ L'antre des nymphes coryciennes, rendu célèbre par les fouilles de l'École française, se taille une part de choix dans l'ensemble des occurrences du mot (5) et d'autres nymphes, sphragidiennes, trouvent également refuge dans un

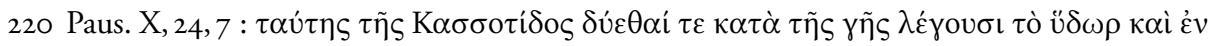

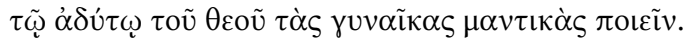

22I Paus. $X, 24,5$.

222 Cf. G. Daux, Delphes, son oracle et ses dieux (1976) I05-IIо.

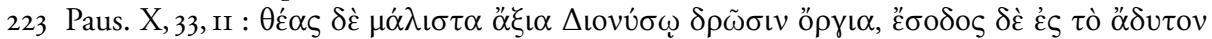

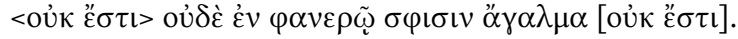

224 I6 occurrences (dont une citation) pour 9 lieux différents, dont 4 sont indépendants d'une description directe.

225 À propos de Clazomènes, Pausanias évoque l'existence d'un antre " de la mère de Pyrrhos " et une histoire locale sur le berger Pyrrhos (VII, 5, II).

226 Paus. V, I9, 6.

227 Paus. III, 24, 4 .

228 Paus. VIII, 42, 6 .

229 Paus. VIII, 42, I. 
antre, sur le Cithéron. ${ }^{230}$ Toujours en Béotie, à Lébadée, une grotte vouée à des dieux guérisseurs est appelée antron dans le récit mythique de sa découverte. ${ }^{231}$ Pausanias mentionne également deux antres de Phrygie ${ }^{232}$ dont la description intervient parmi celle d'autres grottes remarquables. Toute grotte vouée à un dieu ne reçoit cependant pas systématiquement le nom d'ö $\alpha \tau$ cov dans la Périégèse ${ }^{233}$ et le mot n'est pas limité à la désignation d'un sanctuaire.

\subsection{MÉ $\gamma \alpha \rho \nu^{234}$}

Le megaron ancien est une vaste salle du palais mycénien comportant un foyer, parfois l'appartement des femmes. ${ }^{235}$ Hormis les deux extraits des Ehoai que cite Pausanias, ${ }^{236}$ ce sens archaïque n'apparaît pas dans le texte.

Au livre I, parlant du toponyme Méy $\alpha \rho \alpha$, Pausanias se lance dans une explication étymologique du nom de la cité. C'est sous le règne du roi Kar que, pour la première fois, apparurent des hiera de Déméter et que les hommes usèrent du nom de Megara. ${ }^{237}$ Que le $\mu$ ć $\propto \rho \rho v v$ soit bien, à l'origine, un sanctuaire de Déméter, dans l'esprit du Périégète, est confirmé lors de la description de l'acropole Karia

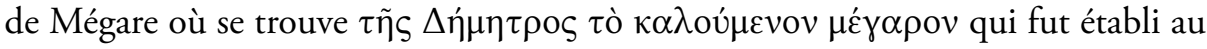
temps du roi éponyme de la citadelle. ${ }^{238}$ Quant à savoir quelle réalité topographique ou architecturale une telle appellation recouvre, Pausanias n'en offre guère la possibilité, pas plus à Mégare qu'ailleurs. L'évocation du megaron de la déesse à Kainèpolis en Laconie ne dépasse pas le simple énoncé, ${ }^{239}$ mais la juxtaposition d'un naos d'Aphrodite laisse entendre que l'édifice pour Déméter n'est pas identifiable à un « temple" au sens strict.

L'apparence des megara attribués à d'autres dieux n'est pas plus claire. Les Méliastes de Dionysos célèbrent les orgia du dieu dans la campagne de Mantinée ; une fontaine porte leur nom et près d'elle se trouve un megaron de Diony-

230 Paus. IX, 3,9 .

23I Paus. IX, 39, 2.

232 Paus. X, 32, 3-4.

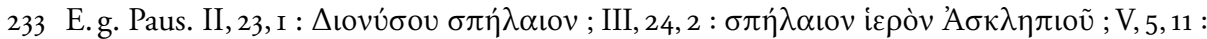

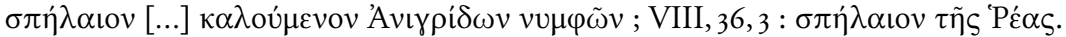

234 II occurrences, dont 2 citations des Ehoai et une restitution. 7 occurrences relèvent d'une description pour 6 endroits différents.

235 P. Chantraine, Dictionnaire étymologique de la langue grecque (1968) 674.

236 Paus. IX, 36, $7 ; 40,6$.

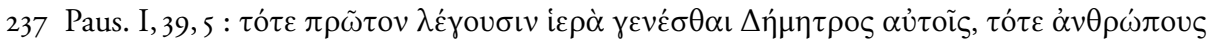

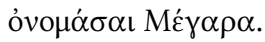

238 Paus. I, 40, 6.

239 Paus. III, 25, 9 : $\dot{\varepsilon} v \alpha \hat{v} \tau \tilde{\eta} \delta \grave{\varepsilon} \mu \dot{\varepsilon} \gamma \alpha \rho o v \Delta \eta \dot{\eta} \mu \eta \tau \rho o \varsigma[\ldots]$. 
sos, ${ }^{240}$ sans plus de précision. Les Kourètes de Messène possèdent un megaron et " là on fait se consumer des animaux de toutes sortes ». ${ }^{241}$ Un sacrifice particulier est également en vigueur dans le megaron du sanctuaire de Despoina à Lykosoura, ainsi que la célébration des mystères. ${ }^{242}$ Dans ce dernier cas, le megaron devait être une structure bâtie d'envergure puisqu'il fallait y rassembler les mystes à l'abri des indiscrétions. Les fouilles à Lykosoura ont mis au jour, en un lieu conforme à la description de Pausanias, ${ }^{243}$ une construction - sans doute à ciel ouvert - dont la complexité pourrait expliquer que, les deux fois où il l'évoque, Pausanias le qualifie de $\kappa \alpha \lambda$ oú $\mu \varepsilon v o v \mu \varepsilon ́ \gamma \alpha \rho \circ v$.

Le terme de megaron ne semble donc pas désigner une disposition topographique systématique ou un édifice "canonique ", même s'il apparait dans le cadre de cultes à vocation mystérique ou orgiastique. Dans le cas des Kourètes de Messène et de la Despoina de Lykosoura, un sacrifice hors norme implique une mise en évidence du foyer qui constitue le cœur de la procédure. Serait-ce également cette disposition qui caractérise les différentes occurrences d'un megaron pour Déméter et celui des Méliastes de Dionysos ? On peut au moins en faire l'hypothèse, qui a le mérite de renvoyer au foyer central du megaron dans son acception ancienne. Elle trouve également un écho dans un fragment d'Ammonios de Lamptres : $\tau$ ò $\delta \dot{\varepsilon}$

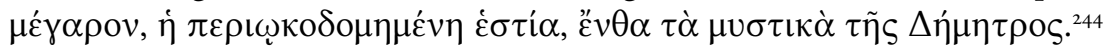

Une seule fois, Pausanias évoque la précipitation des porcelets «dans ce que l'on appelle des megara " ${ }^{245}$ dans le cadre du culte de Déméter et Korè à Potniai en Béotie, sans doute en contexte thesmophoriaque. ${ }^{246}$ Dans ce cadre précis, le terme de megaron s'est substitué à celui de bothros. ${ }^{247}$ Mais les megara architec-

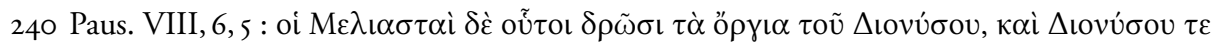

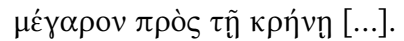

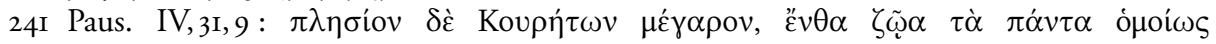

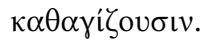

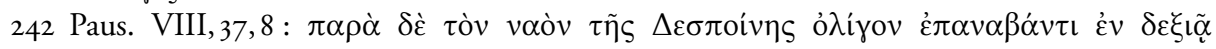

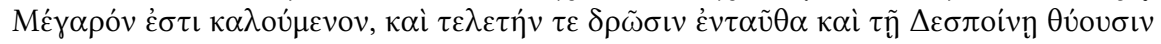

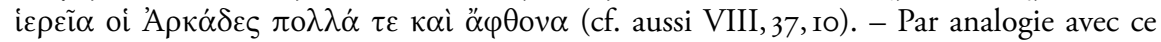
passage, on a restitué un megaron pour l'initiation dans la description du sanctuaire des Grandes déesses à Mégalopolis (VIII, 3I, 7).

243 Cf. Jost, Sanctuaires, op. cit. (note 2) I77, qui argumente en faveur d'une telle identification.

244 A. Tresp, Die Fragmente der griechischen Kultschriftsteller (19I4) 90-96, fr. I. Cf. L. Robert, "Sur deux inscriptions grecques", in : Mélanges Bidez (Annuaire de l'Institut de Philologie et d'Histoire orientales) 2 (1934) 793-8I2 (= Opera Minora Selecta II [I969] 988-IO07), spéc. 8IO-8I2.

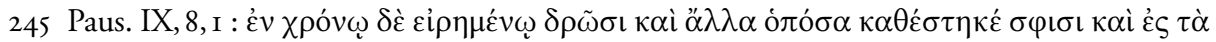

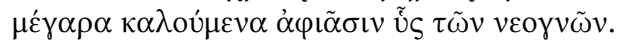

246 W. Burkert, Greek Religion (1985) 242-243.

247 Le mot bothros peut être qualifié de « technique » et désigne un trou, une fosse qui accueille un rituel particulier. Les bômoi portent en hauteur les parts sacrificielles. Le mouvement du 
turaux que croise Pausanias ne sont pas assimilables, ipso facto, à des structures souterraines. ${ }^{248}$

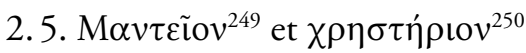

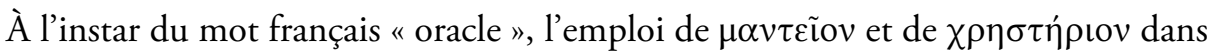
la Périégèse mêle les notions de "sanctuaire oraculaire " et d' "instance oraculaire ". Ces deux termes ne recouvrent pas, en revanche, le troisième sens du mot fran-

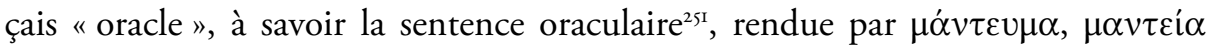
et $\chi \rho \eta \sigma \mu o ́ s .{ }^{252}$ L'oracle de Delphes se taille la plus grande partie des emplois de

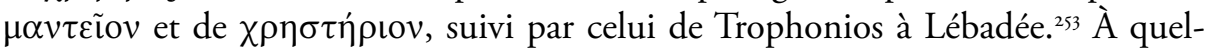
ques reprises, des préoccupations stylistiques ont conduit Pausanias à utiliser les

bothros est inverse, s'enfonçant dans les profondeurs du sol pour en atteindre les puissances spécifiques. Les bômoi sont récurrents dans les descriptions de Pausanias ; les bothroi sont des curiosités rares. À Argos, c'est un certain Nikostratos qui a instauré le rituel du bothros et, à l'époque de Pausanias, les Argiens y jettent encore des torches enflammées en l'honneur de Korè (II, 22,3). À Olympie, dans le temenos de Pélops, le sacrifice d'un bélier noir a lieu au-dessus d'un bothros $\left(\mathrm{V}, \mathrm{I}_{3}, 2\right)$. À Lébadée, à l'endroit où Trophonios a été englouti, se trouve le bothros dit "d'Agamédès ». Ce détail ressort du récit des mésaventures du héros et de son frère Agamédès (IX, 37,7), et le bothros réapparaît lors de la description minutieuse des procédures de consultation (IX, 39, 6). À Titanè, un bômos des Vents accueille une nuit par an un sacrifice, tandis que quatre bothroi sont le lieu d'un rite secret pour se concilier ces dieux, accompagné de formules magiques " de Médée " (II, I2, I).

248 Robert, "Sur deux inscriptions", loc. cit. (note 244) 8IO-8I2, évoque une construction cultuelle qui était, en tout ou en partie, une crypte. A. Henrichs, "MÉy $\alpha \rho o v$ im Orakel des Apollon Kareios ", Zeitschrift für Papyrologie und Epigraphik 4 (1969) 31-37 est plus circonspect. Pour E. Volanaiki-Kontoleontos, qui analyse soigneusement la documentation archéologique et épigraphique sur le thème et fournit un status quaestionis auquel je

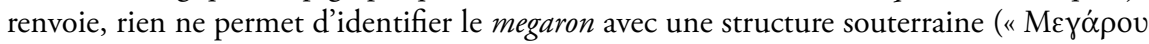

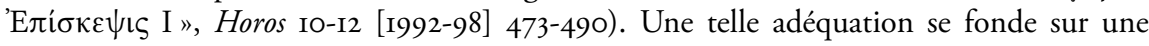
extension abusive de l'image des fosses que doivent être les megara thesmophoriaques.

249 3I occurrences pour II oracles différents.

25016 occurrences (+ I citation) pour 7 oracles différents.

25I Une exception pour $\chi \rho \eta \sigma \tau \eta \dot{\rho} \rho$ เov en $\mathrm{X}, 15,3$.

252 Respectivement 50, 24 et 57 emplois.

253 Manteion de Delphes : I, 2, 5 ; III, I, 6 ; 4, 6 ; IO, 4 ; VIII, 24, 8 ; X, 5, 5-7 (5 occ.) ; 6, 6 ; 24, 4 ; chrèstèrion de Delphes : III, 4, 4 ; II, 8 ; VII, I, 8 ; 2, I ; I9, 4 ; X, 5, 5 ; I3, 8 ; 22, I2. - Manteion de Lébadée : IX, 39, 4-9 (5 occ.) ; 40, I-2 (3 occ.) ; chrèstèrion de Lébadée : IV, 32, 5 ; IX, 40, 2. - Manteion d'Amphilochos en Cilice $(\mathrm{I}, 34,3)$; de la Nuit à Mégare (I, 40,6) ; d'Ammon en Libye (III, I8,3; V, I5, II ; VI, 8,3); d'Ino à Thalamai (III, 26, I) ; de Gè à Olympie $(\mathrm{V}, \mathrm{I} 4, \mathrm{IO})$; d'Apollon à Didymes (VII, 2, 6); d'Apollon à Claros (VII,3, I) ; de Déméter (ou Gè ?) à Patras (VII, 2I, I2); d'Apollon Ptoïos (IX, 23, 6). - Chrèstèrion de Dodone (VII, 2I, 2) ; d'Apollon en Lycie (VII, 2I, I3) ; d'Hermès à Pharai (VII, 22, 2) ; d'Apollon à Claros (VIII, 29, 4); d'Apollon à Abai (X, 35, I). 
deux termes en alternance pour évoquer Delphes et Lébadée, ${ }^{254}$ et l'on ne peut que constater la parfaite synonymie de ce couple de mots dans son texte.

L'ambivalence de la notion d'" oracle " provient de l'ancrage local des instances divinatoires évoquées par manteion et chrèstèrion. Les sentences ou les signes éclairants des dieux sont délivrés dans un sanctuaire, mais rien ne permet d'en déduire que les deux termes font référence à une forme architecturale ou à un complexe naturel déterminés. Manteion et chrèstèrion désignent avant tout l'«institution " oraculaire associée à un endroit donné, ce qui explique pourquoi Pausanias peut se permettre de qualifier un manteion d'ó $\psi \varepsilon v \delta \delta \dot{\varepsilon}^{25 s}$ ou d'en faire le sujet d'une action. ${ }^{256}$

Un exemple de l'emploi de chacun des termes devrait permettre d'en comprendre davantage encore la portée et l'ambiguïté. À Lébadée, où Pausanias s'est lui-même soumis aux procédures oraculaires, le parcours de sa visite est tout entier orienté vers le manteion dont il décrit, d'une part le mode de consultation et, d'autre part, l'apparence formelle, rassemblant dès lors sous une même appellation l'idée de révélation et celle de lieu. ${ }^{257}$ Sur l'agora de Pharai, Pausanias a vu un pilier hermaïque d'Hermès barbu portant l'épiclèse d'Agoraios. Et il enchaîne, « à côté de lui est établi un chrèstèrion ". Il s'agit en fait d'un foyer en pierre situé devant la statue et auquel sont scellées deux lampes en bronze. S'ensuit alors la description du rituel oraculaire. La notion de chrestèrion mêle à la fois la donnée concrète de l'hestia sur laquelle le consultant brûle de l'encens et la notion abstraite d'instance oraculaire à laquelle préside la statue d'Hermès. ${ }^{258}$ En d'autres sanctuaires, le manteion ou le chrèstèrion est caractérisé par une source, ${ }^{259}$ une béance géologique ${ }^{260}$ ou par la conviction que les rêves faits en un tel lieu offrent une réponse aux questions. ${ }^{261}$

L'utilisation du vocabulaire du sanctuaire dans la Périégèse répond généralement à des critères rigoureux. Pausanias ne sacrifie pas volontiers, semble-t-il, la précision d'une désignation à des impératifs de style. ${ }^{262} \mathrm{Il}$ n'en reste pas moins que l'utilisa-

254 Paus. III, 4, 4 et 6 ; IO, 4; II, 8 ; IX, 40, 2 ; X, 5, 5 .

255 Paus. I, 34, 3 ; VII, 2I, I2 ; IX, 23, 6.

256 Paus. I, 2, 5 .

257 Paus. IX, 39, 4-5; 39, 9 .

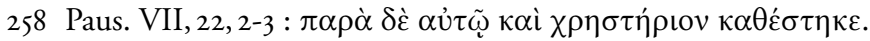

259 Paus. VII, 2I, I2.

260 Paus. IX, 39, $9 ; 40,2$.

26I Paus. III, I8, 3.

262 Voir aussi M. Moggi, "Il lessico del paesaggio in Pausania ", in : P. Radici Colaci (éd.), Atti del II seminario internazionale di Studi sui lessici tecnici greci e latini, Messina, I4-I6 dic. I995 (1997) 189-205, spéc. 204-205. 
tion fréquente du mot ífóv dans une perspective générique ne permet pas, dans bon nombre de cas, de concevoir la réalité topographique que dissimule le mot.

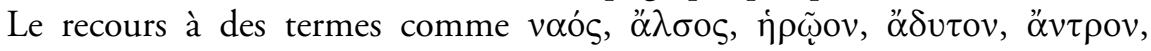

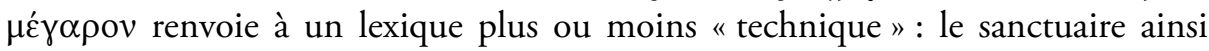
dénommé, en tout ou en partie, comporte l'un ou l'autre élément particulier ou se signale globalement par sa forme singulière. Mais une telle "technicité » reste toute relative dans des descriptions souvent allusives. Des réalités diverses peuvent se cacher derrière un label de ce type. Ainsi, le voós renvoie à la notion générique de temple, dont l'utilisation implique une structure architecturale spécifique - c'est le $v \alpha o \tilde{v} \sigma \chi \tilde{\eta} \mu \alpha$ de certaines tombes -, mais cette donnée n'est pas pour autant " canonique ". L'”̋ $\lambda \sigma o \zeta$ implique un cadre arboré et sacralisé, mais il est des cas où la description semble appeler une désignation en $\alpha \lambda \sigma \sigma \varsigma$ que Pausanias n'utilise pas dans le contexte apparemment adéquat. Le ì $\rho \tilde{\omega} o v$, quant à lui, est l'appellation générique des aménagements entrepris autour d'un tombeau héroïque : le terme est donc technique, mais les réalités concrètes auxquelles ils renvoient sont sans doute plus complexes et plus variées que ne laisse entendre l'usage un peu mécanique du mot par Pausanias. L'" $\alpha \delta$ $\delta$ ov renvoie, lui, à un espace souterrain, quel qu'il soit, et

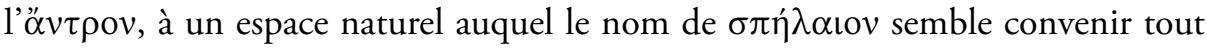

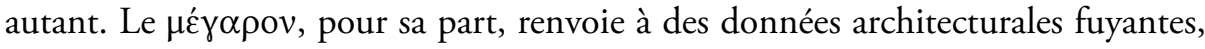
mais le plus petit commun dénominateur des occurrences du terme pourrait être, comme l'affirmait Ammonios de Lamptres, l'existence d'un foyer à la structure monumentalisée.

Les cas où apparait un $\tau \dot{\mu} \mu \varepsilon v o \varsigma$ renvoient essentiellement - mais pas uniquement - à un espace non construit qui tient lieu de «domaine " dévolu à un dieu ou à un héros. Ce sens est également perceptible quand un bâtiment profane passe dans le domaine du culte. D'autres occurrences de temenè divins devaient relever de l'apparence des lieux : il y a fort à parier que le sanctuaire delphique d'Apollon reçoit l'appellation de temenos au vu de la situation particulière de ce vaste ensemble foisonnant qui devait contraster fortement, au temps de sa splendeur, avec l'âpre flanc montagneux du Parnasse.

Les conclusions qu'il est possible de tirer de ce parcours lexical restent certes limitées, et un usage rigoureux de son vocabulaire par Pausanais ne signifie pas que le lecteur moderne puisse systématiquement postuler une même réalité derrière un même mot. De plus, invoquer les nécessités de style pour expliquer nos incompréhensions n'apporte guère d'explication satisfaisante dans bon nombre de cas. En appeler à une logique religieuse dont Pausanias ne ferait pas clairement état semble tout aussi peu convaincant. 
Quoi qu'il en soit, si un tel parcours permettait aux utilisateurs de la Périégèse d'y voir un peu plus clair sur tel ou tel emploi, en donnant d'emblée une vision d'ensemble de tel champ lexical, le présent article aurait atteint son objectif.

Dr. Vinciane Pirenne-Delforge

F. R. S. - FNRS (Université de Liège)

Département des Sciences de l'Antiquité

7, place du 20-Août

$\mathrm{BE}-4000$ Liège

Belgique

v.pirenne@ulg.ac.be 